\title{
Opto-Mechanical Photonic Crystal Cavities for Sensing Application
}

\author{
Ji Xia $\unrhd$, Qifeng Qiao, Guangcan Zhou, Fook Siong Chau and Guangya Zhou * \\ Micro and Nano System Initiative, Department of Mechanical Engineering, Faculty of Engineering, \\ National University of Singapore, 9 Engineering Drive 1, Singapore 117575, Singapore; \\ e0267876@u.nus.edu (J.X.); e0204977@u.nus.edu (Q.Q.); e0115906@u.nus.edu (G.Z.); \\ mpechau@nus.edu.sg (F.S.C.) \\ * Correspondence: mpezgy@nus.edu.sg; Tel.: +65-6516-1235
}

Received: 31 August 2020; Accepted: 5 October 2020; Published: 12 October 2020

\begin{abstract}
A new class of hybrid systems that couple optical and mechanical nanoscale devices is under development. According to their interaction concepts, two groups of opto-mechanical systems are summarized as mechanically tunable and radiation pressure-driven optical resonators. On account of their high-quality factors and small mode volumes as well as good on-chip integrability with waveguides/circuits, photonic crystal $(\mathrm{PhC})$ cavities have attracted great attention in sensing applications. Benefitting from the opto-mechanical interaction, a PhC cavity integrated opto-mechanical system provides an attractive platform for ultrasensitive sensors to detect displacement, mass, force, and acceleration. In this review, we introduce basic physical concepts of opto-mechanical $\mathrm{PhC}$ system and describe typical experimental systems for sensing applications. Opto-mechanical interaction-based $\mathrm{PhC}$ cavities offer unprecedented opportunities to develop lab-on-a-chip devices and witness a promising prospect to further manipulate light propagation in the nanophotonics.
\end{abstract}

Keywords: photonic crystal cavity; opto-mechanical interaction; micro-electro-mechanical systems (MEMS); cavity optomechanics; radiation pressure; optomechanical coupling; sensors

\section{Introduction}

One of the challenging technologies in optics and photonics is the manipulation of light propagation, and it has a direct effect on optical communications, sensing and imaging $[1,2]$. Micro/nano-opto-electro-mechanical systems (M/NOEMS) offer unprecedented opportunities to control the flow of light in nanophotonic structures with efficient and compact architecture, high operation speed and low power consumption [3]. Numerous research works have been developed to establish methods to dynamically control the interaction between photonics and mechanics [4]. Research on opto-mechanical interaction is attracting considerable interests due to its potential for highly efficient and low crosstalk for signal transduction.

For opto-mechanical interaction-based sensing applications, mechanical displacement arising from perturbated factors can be converted into optical transmission signals. One of the general laws of electromagnetism to dynamically control photonic devices is achieved by displacing the dielectric boundaries, also known as mechanically tunable photonics [5]. It has been proved that both the mechanical displacement of dielectric boundary or variation of refractive index (RI) has a crucial effect on the cavity resonance, thus they can serve as a sensitive tool to measure mechanical factors [6]. Electrical actuation is widely implemented by exerting an electrostatic force to obtain moving boundaries or deforming structures, and it has led to the development of M/NOEMS integrated photonic devices. Current on-chip M/NOEMS for photonic integrated circuits (PICs) 
have many competing edges such as a small footprint, easy fabrication process and compatibility of integration with PIC integration [7]. For the purpose of sensing application, with the advantages of flexible mechanical configurations and integration with nano-/micro-electromechanical systems (N/MEMS), mechanically tunable photonic crystal (PhC) cavities have widely developed into a variety of sensors, such as displacement [8-10] and stress sensors [11,12], magnetic [13,14] and electric field [15] sensors. In addition, miniaturization of tunable photonics, which localizes light into sub-wavelength mode volumes in optomechanical resonators, has reinforced the interplay between photons and mechanics to an unprecedented level $[16,17]$. As light exerts forces, radiation pressure from the light reflected from an optical resonator can induce mechanical movement of device. This mechanical displacement, in turn, can change the optical modes of cavity and induce dynamically optomechanics phenomena. Unlike MOEMS tunable photonics under electrical driving, cavity optomechanics enables opto-mechanical interaction by the radiation pressure of light. Some milestone experiments have demonstrated, including ground-state cooling $[18,19]$ and other quantum mechanical phenomena [17]. Understanding the behaviors of radiation force-driven interaction beyond the standard quantum limit [20], cavity optomechanics is also exploited in engineering metrology for fine control of nanophotonic optomechanical devices for sensing applications [21,22].

Microcavities in opto-mechanical interactions consist of Fabry-Perot (F-P) cavities [23], whispering gallery mode (WGM) cavities [24] and photonic crystal (PhC) cavities [25]. Among typical cavities, the $\mathrm{PhC}$ cavity has been demonstrated as an outstanding platform due to its high Q-factors and small mode volume (ultra-high Q/V) [26]. Besides, with good on-chip compatibility with PICs, PhC cavity facilitates the enhancement of opto-mechanical interaction. For the past decade, $\mathrm{PhC}$ cavities based on opto-mechanical interaction have developed quickly, and there have already been many reviews, but they mainly focus on PhC cavity sensing applications [27-30], cavity optomechanics [17,24,31-34] or their applications $[21,22,35,36]$. In this review, we pay attention to the work on sensing application of on-chip PhC cavities based on opto-mechanical interaction. The structure of the review is organized as follows. Section 2 summarizes the experimental configuration of opto-mechanical $\mathrm{PhC}$ cavities. In Section 3, we introduce sensing mechanisms of mechanically tunable PhC cavities and outline the applications of PhC sensors on mechanical sensing. In Section 4, we briefly present the basics of optomechanics phenomena and review the current development of radiation pressure-driven $\mathrm{PhC}$ cavities for sensing applications. Besides, some potential sensing applications integrated with nonlinear mechanics and two-dimensional materials are presented. Finally, we summarize a brief conclusion and discuss the future of opto-mechanical sensing technologies.

\section{Photonic Crystal Cavities for Opto-Mechanical Interaction}

\subsection{Photonic Crystal Cavities}

Optical cavities are capable of confining light based on the RI difference between photonic resonator and its dielectric surroundings. Light circulating inside the photonic cavities can result in an increased effective optical path distance to enhance sensing performance. PhC cavities consist of functional periodic dielectric structures with a periodic variation in the dielectric constant. The periodic dielectric structure gives rise to photonic pass and stop bands, which strongly affects the propagation of electromagnetic waves. Light with frequencies propagating through the periodic structures are called modes. Generally, PhC cavities are established by introducing defects into the cavities, including point or line defect in the periodic structure to create a microcavity or waveguide. From the view of refractive index distribution, $\mathrm{PhC}$ cavities can be broadly divided into three types: one-dimensional (1D), two-dimensional (2D), and three-dimensional (3D) structures [37,38]. Their typical configurations are shown in Figure 1. One-dimensional PhC cavities (see Figure 1a), also called nanobeam cavities, are composed of two symmetrical PhC Bragg mirrors and taper air holes. These two Bragg mirrors can give rise to an F-P-like resonator, which makes Q-factors of $1 \mathrm{D}$ PhC cavities over $10^{6}$ [39]. Inducing defects inside a planar photonic crystal gives rise to $2 \mathrm{D} \mathrm{PhC} \mathrm{cavities.} \mathrm{The} \mathrm{line} \mathrm{defect}$ 
in a $2 \mathrm{D} \mathrm{PhC} \mathrm{(see} \mathrm{Figure} 1 \mathrm{~b}$ ) is called a waveguide and allows the propagation of certain frequencies within the bandgap. Currently reported 2D PhC cavities based on silicon-on-insulator (SOI) material can have Q-factors reaching up to $10^{6}$ [40]. In 3D PhCs, a complete photonic bandgap can be obtained by modulating RI distribution in total three directions. By carefully placing a defect at the lattice center (see Figure 1c), cavity modes can be achieved [41]. Considering the existing nanofabrication technologies with planar lithography, 1D and 2D cavities can be easily achieved owing to their planar structure. In contrast, 3D cavities are difficult to be fabricated in practice. Therefore, we mainly focus on the recent research works based on $1 \mathrm{D}$ and $2 \mathrm{D} \mathrm{PhC}$ cavities in this review.

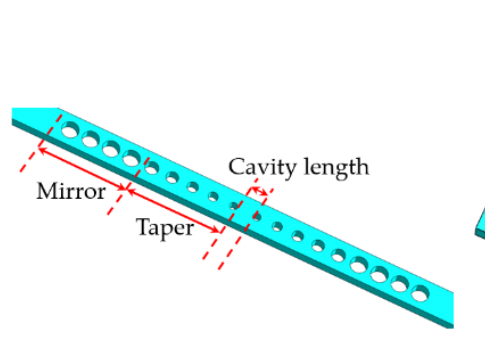

(a)

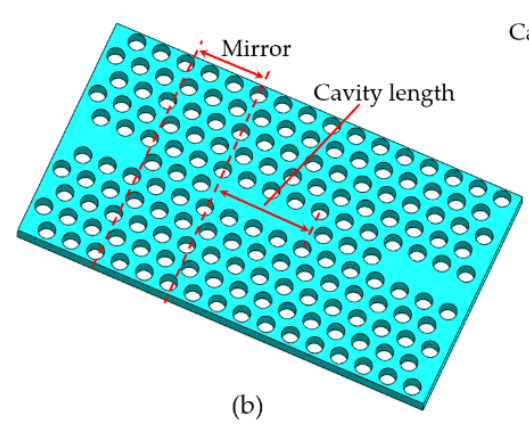

(b)

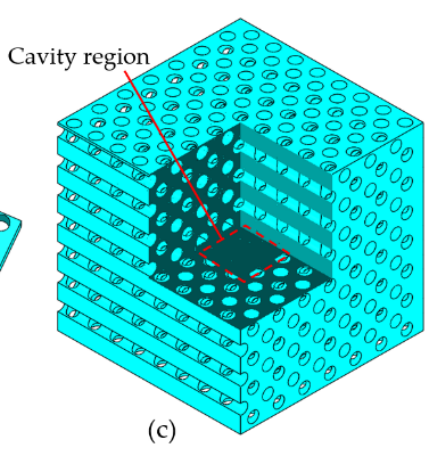

(c)

Figure 1. Schematics of (a) 1D, (b) 2D, and (c) 3D photonic crystal (PhC) cavities [37,38].

\subsection{Experimental Opto-Mechanical PhC Cavities}

Leveraging nanofabrication technologies, optical and mechanical degrees of freedom can be coupled in the nanoscale opto-mechanical system. For the purposes of sensing applications, the on-chip opto-mechanical system is designed to convert variations of sensing factors to the nano-mechanical motion. Furthermore, the nano-mechanical motion can be optically detected via the induced opto-mechanical interaction, thereby probing the target sensing signal. Based on interaction effects between optical cavity and mechanical resonator, opto-mechanical cavities can be divided into two types: mechanically tunable cavities and radiation pressure-driven cavities. Before elaborating the working mechanism of opto-mechanical sensors, we introduce the several typical configurations of optical cavity first.

\subsubsection{One-Dimensional Photonic Crystal Nanobeam in Opto-Mechanical System}

For 1D photonic crystal nanobeam (PCN) cavities, their opto-mechanical configurations and metrics are illustrated and summarized in Table 1. 1D PCN cavities attract great attention for their ultra-compact size, ultra-small mode volume, and high integrability with PICs in the ultra-sensitive optical sensing. A single PCN cavity in the mechanically tunable method was mainly developed to detect an external nanoscale probe driven by a NEMS comb-drive actuator [8,42]. However, mechanical tuning of a probe perturbation inevitably caused a significant drop in Q-factor and transmission of cavity. In the radiation pressure-driven applications, single PCN cavities have been extensively developed for optomechanical systems. To enhance the mechanical quality factor in the suspended PCN cavity, an attached phononic shield-a lattice around the resonator [43] was proposed. The lattice phononic shield enhanced the arbitrary mechanical modes of a nanobeam by adjusting the frequency of phononic band gap, which reduced mechanical loss while maintaining the optical properties of cavity mode. 
Table 1. Typical configurations of 1D PhC cavities for opto-mechanical interactions.

\begin{tabular}{|c|c|c|c|c|c|c|}
\hline \multirow[b]{2}{*}{ Classification } & \multirow{2}{*}{$\begin{array}{l}\text { Single PCN } \\
\text { Cavity }\end{array}$} & \multirow{2}{*}{$\begin{array}{c}\text { Coupled PCNs } \\
\text { Cavity }\end{array}$} & \multirow{2}{*}{$\begin{array}{l}\text { Zipper } \\
\text { Cavity }\end{array}$} & \multicolumn{2}{|c|}{ Split PCN Cavity } & \multirow{2}{*}{$\begin{array}{c}\text { Slot } \\
\text { Embedded-PCN } \\
\text { Cavity }\end{array}$} \\
\hline & & & & $\begin{array}{l}\text { Longitudinally } \\
\text { Split PCN }\end{array}$ & $\begin{array}{l}\text { Transversely } \\
\text { Split PCN }\end{array}$ & \\
\hline Structure & 00000000000 & 10000000000000 & 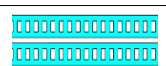 & 00000000000000 & awawar & 000000000000 \\
\hline Optical Q & $9.06 \times 10^{5}$ & $\sim 3.8 \times 10^{3}$ & $\sim 10^{4}-10^{5}$ & $5.2 \times 10^{3}$ & 400 & $\sim 1.2 \times 10^{5}$ \\
\hline $\begin{array}{l}\text { Mechanical Q } \\
\text { Mechanical frequency }\end{array}$ & $6.8 \times 10$ & & $50-150$ (air) & $<100$ (air) & N/A & 417 (air) \\
\hline$\left(\Omega_{m} / 2 \pi\right)$ & $5.1 \mathrm{GHz}$ & $\sim 1.18 \mathrm{MHz}$ & $\sim 8 \mathrm{MHz}$ & $10.5 \mathrm{MHz}$ & $\sim 3 \mathrm{MHz}$ & $2.69 \mathrm{GHz}$ \\
\hline $\begin{array}{l}\text { Optomechanical } \\
\text { coupling rate }\end{array}$ & $\begin{array}{c}1.1 \mathrm{MHz} \\
(\mathrm{g} / 2 \pi)\end{array}$ & $\begin{array}{l}\sim 6.43 \mathrm{kHz} \\
(\mathrm{g} / 2 \pi)\end{array}$ & $123 \mathrm{GHz} / \mathrm{nm}$ & $3 \mathrm{GHz} / \mathrm{nm}$ & $\begin{array}{c}11.5 \mathrm{MHz} \\
(\mathrm{g} / 2 \pi)\end{array}$ & $\begin{array}{c}310 \mathrm{kHz} \\
(\mathrm{g} / 2 \pi)\end{array}$ \\
\hline Mode volume & N/A & N/A & $0.2(\lambda / n)^{3}$ & $0.35(\lambda / n)^{3}$ & $\mathrm{~N} / \mathrm{A}$ & $0.017(\lambda / n)^{3}$ \\
\hline Mass & $127 \mathrm{fg}$ & $\sim 10 \mathrm{pg}$ & $\sim 43 \mathrm{pg}$ & $\sim 1 \mathrm{pg}$ & $\sim 2.4 \mathrm{pg}$ & $103 \mathrm{fg}$ \\
\hline Reference & [43] & [44] & [45] & {$[46]$} & [47] & [48] \\
\hline
\end{tabular}

When two PCN cavities are placed close to each other and their mode fields are coupled together, resonant frequencies of coupled supermodes can be adjusted by controlling the coupling strength between two cavities. In mechanically tunable coupled PCN configurations, under the applied electrostatic force of N/MEMS actuation, physical displacement of moveable PCN cavity could be modulated in-plane gap between two nanobeams [49-52], in-plane laterally center offset [53,54], out-of-plane rotation of suspended cavity [55] as well as out-of-plane bending nanobeams [56]. In a similar method, a vertically coupled PCN cavity [57] was electrostatically controlled. With two PCNs etched on two vertically coupled GaAs flexible nanobeams, a large wavelength tuning range over $12 \mathrm{~nm}$ was achieved through a bias voltage $15 \mathrm{~V}$. Moreover, triple-coupled PCN cavities [58,59] with a Q-factor reaching up to $8 \times 10^{4}$ have been reported. By precisely controlling the position of cavity vertically and horizontally, a 41.69-pm wavelength tuning of resonance and small degradation of Q-factor were obtained [59]. Coupled resonant system with high Q-factor and high transmission opened interesting perspectives in nonlinear optics and signal processing. In the radiation pressure-driven systems, two laterally coupled PCN cavities $[44,60]$ could support propagating waveguide modes and achieve a large coupling strength to $\mathrm{GHz}$ mechanical resonators in the $\mathrm{Si}_{3} \mathrm{~N}_{4} \mathrm{PCN}$ cavity. More importantly, a flexible design of mechanical resonator could greatly vary mechanical stiffness and frequency, in turn, resulting in stronger optomechanical coupling strength. Another laterally coupled PCNs [45,61-63] also called zipper cavity, were designed and fabricated with resonator mass of pg order of magnitude. Consequently, a combination of decreasing mechanical loss at the mechanical frequency and reduced effective motion mass (10 pg to $50 \mathrm{fg}$ ) resulted in a dramatically enhanced force sensitivity of optomechanical system. In the zipper nanobeams, an optical force-induced spring effect was observed through mechanical frequency shift and linewidth variation. From the perspectives of engineering applications, zipper nanobeam paved the way for wide-bandwidth optical frequency conversion and filtering induced by optical forces. Besides, a vertically coupled PCN cavity [64] was demonstrated with a lower motion mass, and a comparable optical $Q$-factor and mode volume, resulting in larger zero-point fluctuations of mechanical modes and larger photon-phonon coupling rates.

Except for the tunable mechanisms of coupled PCN cavities, another effective approach to tune a cavity is to reshape the resonator itself. Split nanobeam in PCN cavity is a typical example, and it can be structurally classified into longitudinally split and transversely split cavities. The longitudinally split PCN cavity consisted of an air gap in the middle of nanocavity and a pair of Bragg mirrors patterned on side cantilever beams. By altering the axial separation gap of two aligned cantilevers, the Q-factor of the cavity was largely tuned and yet, the resonant frequency was barely affected [65]. Longitudinally split PCN cavities could also be mechanically controlled by varying the offset laterally in-plane [66] and out-of-plane [67] while maintaining the constant separation gap. The transversely split PCN cavity was split by an air-slot into two symmetric parts. By controlling both movable structures by the comb-drive actuators, the width of air-slot affected the performance of cavity resonance. In the reported experiments $[68,69]$, the second-order mode resonance shift of up to $17 \mathrm{~nm}$ was achieved with an air-slot 
gap increment of $26 \mathrm{~nm}$ while the Q-factor could be maintained. In the radiation pressure-driven systems, the dynamics of suspended structure in the longitudinally split PCN cavities [46,70] were applied for detecting mechanical motions. Likewise, the transversely split PCN cavity mechanically resembled a pair of double clamped beams [47], and the half beam with an ultra-small mass ( 2.4 pg) and small spring constant contributed to a large mechanical motion with an ultra-high optomechanical coupling rate $g_{o} / 2 \pi \approx 11.5 \mathrm{MHz}$. To further enhance optomechanical coupling, a central nano-slot was added in the middle of the PCN cavity [48,71]. In Ref. [48], the slotted PCN optomechanical cavity with an ultrasmall mode volume $0.017(\lambda / n)^{3}$ and an optical Q-factor up to $1.2 \times 10^{5}$ was demonstrated, which enabled the device to work in the resolved sideband with an optomechanical coupling rate of $310 \mathrm{kHz}$ and a measured mechanical mode of $2.69 \mathrm{GHz}$. In such a configuration, the maximum electric field in the small mode volume was localized out of dielectric material. It provided an attractive platform to explore an additional coupling to the mechanical resonator (e.g., carbon nanotubes and nanowires).

\subsubsection{Two-Dimensional Photonic Crystal Cavity in Opto-Mechanical System}

For 2D PhC cavities, mechanical tuning effects on the cavity resonance can also be classified based on the working mechanisms of probe perturbation, deformable cavity and coupled cavities, as outlined in Table 2. First, the shape of planar 2D PhC slab cavity in the mechanical tunable system can be modulated under the applied mechanical forces. Lee et al. [72,73] investigated the property of a ring 2D PhC cavity based on the stress-induced deformation of microcantilever. When an external force was applied on the cantilever tip, holes inside the PhC cavity were deformed, thereby resulting in the resonance shift. In addition to the cavity deformation, the presence of probe perturbation could alter the resonance of 2D PhC slab cavity. For example, an electrostatically driven bimorph probe was self-aligned to the holes of $2 \mathrm{D} \mathrm{PhC}$ cavity, so the propagation properties of $\mathrm{PhC}$ cavity could be altered based on the proximity of tips to holes [74]. The presence of a probe accounted for a variation of effective RI in 2D PhC cavity, thereby mechanically modulating the photonic band structure of the cavity. Second, a tunable air-slot 2D PhC slab was developed with in-plane motion driven by an integrated MEMS actuator $[75,76]$. Due to the dielectric discontinuity in the air-slot, optical mode was locally confined and enhanced inside the air-slot gap. Therefore, motion tuning of the width of air-slot gap could exert an intensive impact on the resonance and Q-factor of cavity. Integrating a nanocantilever inside a 2D PhC cavity was numerically and experimentally studied [77,78], wherein the nanocantilever acted as a tunable photonic defect in the 2D PhC slab cavity. Strong opto-mechanical interaction between nanocantilever and defect-mode field resulted in a good sensing performance of resonance dispersion to surface stress-induced out-of-plane deflection. Finally, a vertically coupled bilayer 2D PhC cavity was also experimentally demonstrated $[79,80]$. The electrostatic actuating out-of-plane motion-controlled separation gap between two membranes, as a result, a resonant wavelength tuning range of $13 \mathrm{~nm}$ was obtained at a lower temperature of $8 \mathrm{~K}$ [79].

Table 2. Typical configurations of 2D PhC cavities for opto-mechanical interactions.

\begin{tabular}{|c|c|c|c|c|c|}
\hline Classification & $\begin{array}{l}\text { Planar PhC } \\
\text { Slab Cavity }\end{array}$ & $\begin{array}{l}\text { Air-Slot PhC } \\
\text { Cavity }\end{array}$ & $\begin{array}{c}\text { Cantilever-PhC } \\
\text { Cavity }\end{array}$ & $\begin{array}{l}\text { Slot Embedded } \\
\text { PhC Cavity }\end{array}$ & $\begin{array}{c}\text { Vertically } \\
\text { Coupled Bilayer } \\
\text { PhC Cavity }\end{array}$ \\
\hline \multicolumn{6}{|l|}{ Typical structure } \\
\hline Optical Q & $\sim 10^{4}$ & $>10^{6}$ & $\sim 371$ & $5.3 \times 10^{4}$ & $1.6 \times 10^{3}$ \\
\hline Mechanical Q & 890 & $50-100$ & N/A & $>10^{4}$ & $\sim 2$ (air) \\
\hline Mechanical frequency $\left(\Omega_{m} / 2 \pi\right)$ & $9.54 \mathrm{MHz}$ & $\sim 3 \mathrm{MHz}$ & $\mathrm{N} / \mathrm{A}$ & $>600 \mathrm{MHz}$ & $1.8 \mathrm{MHz}$ \\
\hline Optomechanical coupling rate & $0.23 \mathrm{kHz}(\mathrm{g} / 2 \pi)$ & $152 \mathrm{GHz} / \mathrm{nm}$ & N/A & $>200 \mathrm{kHz}(\mathrm{g} / 2 \pi)$ & N/A \\
\hline Mode volume & $\sim 3(\lambda / n)^{3}$ & N/A & N/A & $\sim 0.021(\lambda / n)^{3}$ & N/A \\
\hline Reference & [81] & [82] & [74] & [83] & {$[80]$} \\
\hline
\end{tabular}


Similarly, optical configurations of 2D PhC cavity in the radiation pressure-driven system are almost same as those in the mechanically tunable system in Table 2. 2D planar PhC cavities can support photonic and phononic band gaps simultaneously. The simplest way is to create an optomechanical structure from a single planar PhC slab [81,84]. In this structure [81], the optical modes of a L3 cavity were strongly coupled to localized mechanical modes, which could be used for exciting photon-phonon conversions. The air-slot PhC structure $[82,85]$ was established by confining the cavity field into the air-slot between two suspended and flexible membranes. This system could activate the dynamic backaction to realize mechanical amplification or cooling. It played a significant role in exploiting sensing platform utilizing optomechanical backaction with reductive thermal noise and low-noise optical read-out. Another nanomechanical slot embedded PhC cavity was achieved by defining three missing holes $[83,86]$. The ultra-small dimension and femtogram mass of nanomechanical resonators with high mechanical and optical Q-factors were promising for ultrasensitive mechanical measurements and mass sensing of nanoparticles.

\subsection{Discussion}

The interactions between an optical and mechanical resonator based on opto-mechanical cavities including 1D and 2D PhC cavities have been experimentally demonstrated with strong optomechanical coupling. In the mechanically tunable methods, N/MEMS actuators are employed to realize the displacement of photonic device in micro/nano scale. Under the applied electrostatic force or external force of N/MEMS actuation, a strong effect on the optical field could react with the displacement of boundaries or probe perturbation. Integration of N/MEMS and PhC cavities open the opportunities for sensing application based on nanoscale opto-mechanical interaction in PhC cavities. In the radiation pressure-driven schemes, both optical and mechanical resonators are fabricated in a nanostructure through periodic patterning, this makes the localized photon-phonon interaction happen in a single device. Compared to the Fabry-Perot cavity and Whispering-Gallery-Mode cavity, PhC cavity has a greatly reduced mechanical mass to the magnitude of a picogram, which is particularly suitable for ultrasensitive nanoparticle sensing using optomechanical backaction effects. Meanwhile, the beam width in PhC cavity approaching to or below the order of light wavelength confines the cavity mode in a nanoscale structure, resulting in a stronger optomechanical coupling than that obtained in F-P or WGM resonators. In addition, a flexible mechanical geometry can be effectively coupled with an optical mode driven by optical force, which provides a perfect platform to observe optomechanical dynamics, e.g., optical spring or damping effects, and to explore various sensing applications.

\section{Mechanically Tunable PhC Cavity Sensors}

Mechanically tunable photonics, i.e., by mechanically moving or deforming the mechanical structures, is a natural way to tune photonic devices. Leveraging the optimization of both optical and mechanical design, the mechanical movement induced by sensing target can be detected through optical readout. Owing to their compact size and high mechanical flexibility, PhC cavities can be applied for mechanical sensing by adjusting the coupling between a light field and nanomechanical motion. In this section, we first briefly introduce sensing mechanisms of mechanically tunable PhC cavities and then present sensing applications based on this opto-mechanical configuration.

\subsection{Sensing Mechanisms of Mechanically Tunable PhC Cavities Sensors}

In PhC cavities, resonance states of optical cavities can be controlled either by varying RI in the given medium, or by moving physical boundaries between media of different indices. In the sensing systems, the induced change of resonance state is typically detected using resonance shifts or varying Q-factors. Here, we first introduce sensing principles of the optical cavity based on perturbation theory [87]. In the waveguide-cavity coupling configurations, the input-output formalisms for optical cavities are outlined in Figure 2a. Figure 2(a-1) presents an evanescently side coupled waveguide-cavity, such as a bus waveguide (WG) coupled into a PhC cavity [88]. In this case, optical fields transmitted 
from resonator are detected and represented in the spectrum (see Figure 2b). Figure 2(a-2) describes a directly double-coupled resonator, where the transmission spectrum has a reversely characteristic peak resonance [89]. Figure 2(a-3) shows a single-side coupled resonator and reflection optical fields are measured in the spectrum [90]. At the optical resonance, light circulates inside the optical resonator and thereby a dip occurs in the transmission spectrum as shown in Figure 2b; Blue, red and dashed red dips in the spectrum denote cavities without any perturbation, with RI perturbation and absorptive perturbation, respectively. Detection of resonant wavelength shift $\delta \lambda$ or linewidth variation $\Delta \lambda^{\prime}-\Delta \lambda$ provides an effective approach to detect the sensing factors arising from RI variation or moving boundary.

(a)

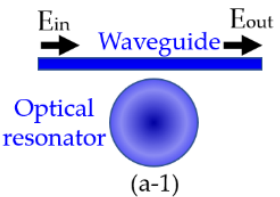

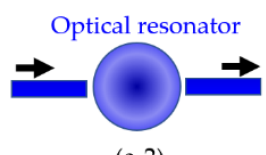

$(\mathrm{a}-2)$

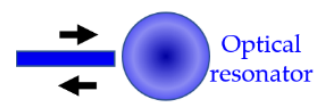

$(\mathrm{a}-3)$

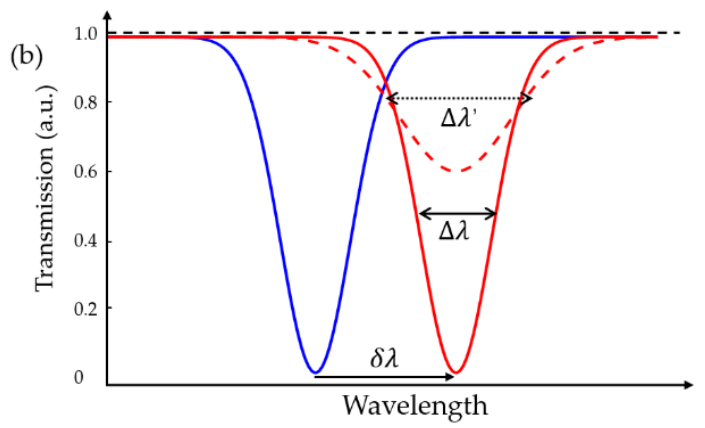

Figure 2. (a) Configurations of waveguide-resonator coupling: (a-1) waveguide (WG) side-coupled [88], (a-2) double side-coupled [89], and (a-3) single side-coupled [90]; (b) a characteristic dip at resonance wavelength for WG side-coupled cavity. The resonance shifts from blue line to red line due to perturbation, and a linewidth broadening $\Delta \lambda^{\prime}-\Delta \lambda$ leads to a decreasing Q-factor [29].

In the mechanically tunable photonics, $\mathrm{PhC}$ cavities have exceptional advantages for sensing application due to their ultra-high $\mathrm{Q} / \mathrm{V}$. Q-factor is expressed as $\mathrm{Q}=\lambda_{\text {cav }} / \Delta \lambda$, which is a ratio of resonant wavelength to its linewidth. High $Q$-factor indicates that a narrower linewidth $(\Delta \lambda)$ occurs at the resonance and determines the minimum detectable resonance shift $(\delta \lambda)$. Mode volume $V$ describes the capability of the light confinement. A small mode volume $V$ represents that the mode field is highly confined inside the cavity, so that it can significantly enhance light-matter interaction. Currently, most sensing applications based on mechanically tunable PhC cavities mainly have three working mechanisms, including dielectric boundary moving, near-field probe perturbation as well as coupled mode-splitting. Deformation or movement of dielectric boundaries, related to the displacement of mode field profile, affects dielectric tensor between different materials. Resonance frequency variation due to the effects of moving dielectric boundary can be given as [91],

$$
\frac{d \omega}{d \xi}=\frac{-\omega_{c a v} \int d V d \varepsilon / d \xi|\boldsymbol{E}(\boldsymbol{r})|^{2}}{2 \int d V \varepsilon|\boldsymbol{E}(\boldsymbol{r})|^{2}}
$$

here, $\xi$ indicates change of the physical boundaries, and the dielectric index $\varepsilon$ is a function of position $\varepsilon=\varepsilon(r)$. A larger energy density $\varepsilon|E(r)|^{2}$ at the moving interface could maximize the interaction between optical and mechanical resonators. Therefore, moving dielectric boundary of resonator plays a significant role in the deformed cavities for sensing application.

Meanwhile, the presence of external dielectric matters also exerts an effect on resonance of PhC cavities. Assuming that the sensing mechanism based on RI variation by monitoring resonance shift upon an arrival event of an analyte, according to the perturbation theory, the resonant frequency shift $\Delta \omega$ induced by a near-field probing analyte is represented by $[87,92]$,

$$
\frac{\Delta \omega}{\omega_{c a v}}=\frac{\alpha_{e x}\left|E\left(r_{p}\right)\right|^{2}}{2 \int \varepsilon|E(r)|^{2} V}
$$


where $\alpha_{e x}$ and $\varepsilon$ describe the polarizability of surrounding analyte and dielectric constant of cavity; and $E(r)$ and $E\left(r_{p}\right)$ are electric field inside cavity and the probing analyte position $r_{p}$; it is obvious that the presence of a dielectric probe analyte can cause a red shift of resonance frequency based on the distance between probe perturbation and cavity. Besides, both a small mode volume $V$ and intensive light confinement in the perturbation area can result in a wide $\Delta \omega$ to achieve a preferable sensitivity. In addition, it should be noted that monitoring Q-factor can also detect an external analyte based on RI perturbation theory. Analytes-perturbated cavity sensor translates change of RI of external analyte into the change of linewidth. In the optical cavity with an approaching probe analyte, optical absorption induced cavity mode linewidth broadening has been widely exploited to probe nanoparticles $[93,94]$.

Moreover, displacing coupled photonic resonators are also widely used in mechanically tunable $\mathrm{PhC}$ sensors, namely coupled PhC cavities. The resonance frequencies of coupled supermodes can be controlled by tuning the coupling strength between two cavities. According to the temporal coupled mode theory (CMT) [95], when two identical PhC resonators are placed close to each other, their mode fields are coupled as below,

$$
\left\{\begin{array}{l}
\frac{d}{d t} a_{1}=j \omega_{c a v} a_{1}-j \kappa a_{2} \\
\frac{d}{d t} a_{2}=j \omega_{c a v} a_{2}-j \kappa a_{1}
\end{array}\right.
$$

where $\kappa$ is coupling strength between two cavities with natural frequency $\omega_{c a v}$; and $a_{1}$ and $a_{2}$ denote the optical modes in their individual resonators. Resonance of coupled cavities can be given as,

$$
\omega_{i}=\omega_{c a v} \pm \kappa
$$

For coupled PCN cavities, the interaction of two cavities causes a splitting of their original uncoupled modes into corresponding pairs of even mode (symmetric mode shape) and odd mode (anti-symmetric mode shape). As can be seen from Equation (4), the coupling strength $\kappa$ determines resonance frequency and it is sensitive to the mechanical displacement between two PCN cavities. Therefore, tuning the mechanical displacement of movable photonic resonator plays a significant impact on the mode-splitting status and it can be easily compatible with an on-chip integrated N/MEMS system for sensing applications.

\subsection{Sensing Applications of Mechanically Tunable PhC Cavities}

On-chip tuning PhC resonator devices have merits of simple system configurations and fabrication processes, dynamic control capability and low energy consumption. When certain mechanical action is applied to PhC cavity, resulting in variation of effective RI perturbation, deformation of resonator as well as mode-splitting in coupled cavities, could strongly affect the transmission spectrum of $\mathrm{PhC}$ cavity. Therefore, the amount of such spectral shift can be exploited to detect the applied mechanical actions. Now, we turn to review the sensing applications based on mechanically tunable PhC cavities.

\subsubsection{Deformable Cavity-Based PhC Sensors}

Under the operation of external physical forces, the deformation of cavity structure can modify the cavity modes and lead to cavity resonance shifts and varying quality factors. Research efforts have been carried out to achieve the mechanical sensing based on mechanically tunable PhC cavities. 1D PCN cavities have attracted great attention in optomechanical sensing owing to their smaller size as well as the higher design flexibility. Split PCN cavity is a typical example of reconfigurable or deformable cavities, as shown in Figure 3. The split PCN cavity consisted of an air-slot in the middle of a nanocavity, which separated the nanobeam cavity into two symmetric parts. The movable side was mechanically controlled by the comb-drive, and the width of air-slot was tuned by varying the voltage applied to the actuator. By changing the axial separation of two aligned cantilevers (see Figure 3b), Q-factor of cavity was largely tuned and yet, while resonant frequency was barely affected [65]. Longitudinally split nanobeam cavities could also be mechanically tuned by varying the offset laterally in-plane (see Figure 3c) [66] and out-of-plane (see Figure 3d) [67] while maintaining the constant separation gap. 
Another ladder-like cavity was transversely split by an air-slot into two symmetric parts, as shown in Figure 3e. By controlling both movable structures by their individual comb-drives, the width of air-slot affected the performance of cavity resonance. In the reported experiments [68,69], a resonance shift of up to $17 \mathrm{~nm}$ of the second-order mode was achieved with an air-slot width increment of $26 \mathrm{~nm}$ while the Q-factor could be maintained. Both these two split PCN cavities were mechanically tuned to deform their optical cavities and then greatly affected optical resonance, therefore tunable configuration based on this split PCN cavity was sensitive to exploit sensing applications on physical displacement, force and vibration etc. Taking a compact and geometrically isolated actuator (also called nanobender) as an example, it composed of metal electrodes on a single layer of piezoelectric thin-film, as demonstrated in Figure 4 [96]. Combined with a sliced $\mathrm{PhC}$ cavity for displacement sensing, a wavelength tunability of an optical resonance by $\sim 5 \mathrm{~nm} / \mathrm{V}$ with a large tuning linewidth ranging from 1520 to $1560 \mathrm{~nm}$ at the applied voltage of $4 \mathrm{~V}$. Additionally, several nanobender-integrated schemes were proposed to detect the displacement induced by physical actuations operating in-plane, out-of-plane and twisting motions [96].
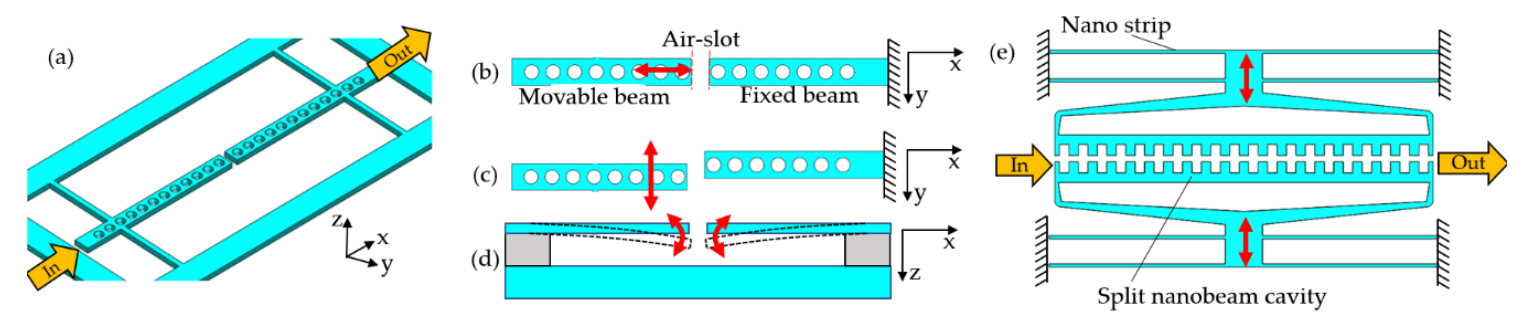

Figure 3. (a) Schematic of tunable longitudinally split PCN cavity; (b) longitudinal motion of side nanobeam [65]; (c) lateral motion of side nanobeam [66]; (d) bending of both sides of nanobeam [67]; (e) schematic of a tunable transversely split PCN cavity [68]. All red arrows indicate the motion direction.
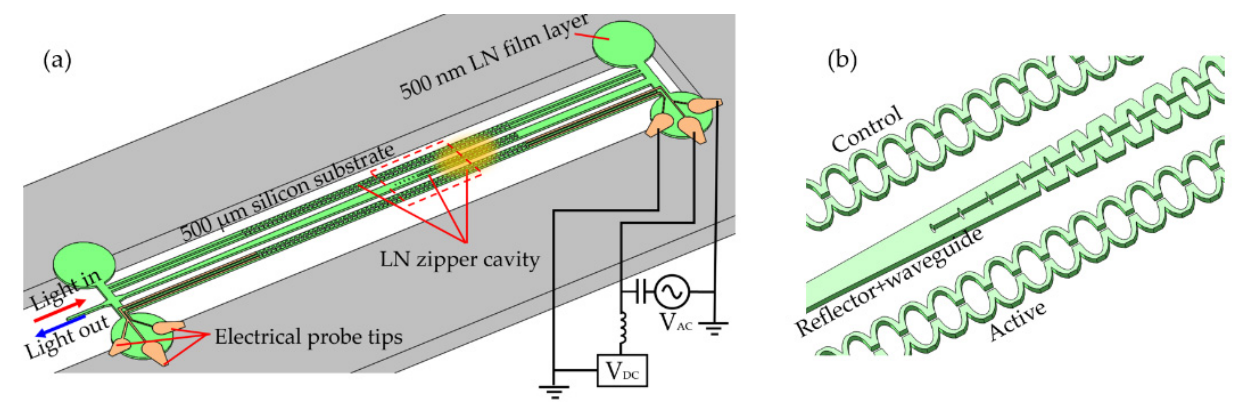

Figure 4. Nanobender cavity for displacement sensing: (a) nanobender cavity with waveguide-zipper $\mathrm{PhC}$ cavities coupling and electrical actuations; (b) close-up view on the zipper PhC cavities and waveguide coupling structure [96].

Besides 1D PhC cavities, reconfigurable cavities can also be more effectively realized with 2D PhC cavities. First, the geometrical deformation of cavity was introduced based on 2D PhC slabs. Lee et al. presented a pressure sensor with a shoulder-coupled PhC cavity located on a suspended silicon bridge [97]. As shown in Figure 5a, when the pressure was applied on the PhC cavity, longitudinal deformation of air holes and defect length could shift the resonant wavelength of PhC cavities. It was demonstrated that the minimum detectable force and vertical deflection reached up to $0.25 \mathrm{~N}$ and $20-25 \mathrm{~nm}$, respectively. Moreover, it pointed out that the relative position variation of air holes in the deformed PhC cavity played a major role in contribution to the behavior of optical resonance. On this basis, Mai et al. investigated a hexagonal nano-ring PhC slab by using a silicon cantilever (see Figure 5b) [73]. A roughly linear relationship between the applied strain and resonance shift was observed. When the ring $\mathrm{PhC}$ was embedded onto a $50 \mu \mathrm{m} \times 15 \mu \mathrm{m}$ planar cantilever, the minimum detectable force and strain for ring $\mathrm{PhC}$ were $75.7 \mathrm{nN}$ and $0.0023 \%$, respectively. Compared with shoulder-coupled PhC configuration [97], this cantilever design greatly enhanced the sensitivity. Later 
in 2011 [72], they further investigated the sensing schematics of dual ring PhCs with various positions close to the junction of cantilever and substrate. The detection limit of force reached up to $7.58 \mathrm{nN}$. Besides, they also proposed a silicon device integrated with triple ring PhCs as a mechanical sensor [11], wherein a minimum detectable force of $0.847 \mu \mathrm{N}$ with a force ranging from 10 to $20 \mu \mathrm{N}$ was obtained. Similar to Lee's measurement system, Tung et al. [98,99] proposed a strain sensor using a silicon 2D $\mathrm{PhC}$ cavity by loading standard weights to the planar cantilever so that longitudinal strains were created in the PhC structure, as shown in Figure 5c. A linear relationship between applied strain and resonance shift was identified with a sensitivity of $0.95 \mathrm{pm} / \mu$-strain. Additionally, an electric field sensor based on reversible strain-tuning of a quantum dot strongly coupled to a PhC cavity [15] was demonstrated in Figure 5d. By mounting PhC cavity sample and piezo-electric actuator in parallel on the holder, the applied stress in the y-axis direction was exerted to deform defect of $\mathrm{PhC}$ cavities. The in-plane strain induced by applied electric field could lead to expansion or contraction of the $\mathrm{PhC}$ defect areas, thereafter the shift of resonant wavelength. Therefore, a redshift of $0.45 \mathrm{~nm}$ was experimentally achieved with an electric field of $15 \mathrm{kV} / \mathrm{cm}$ applied to a piezo-electric actuator.
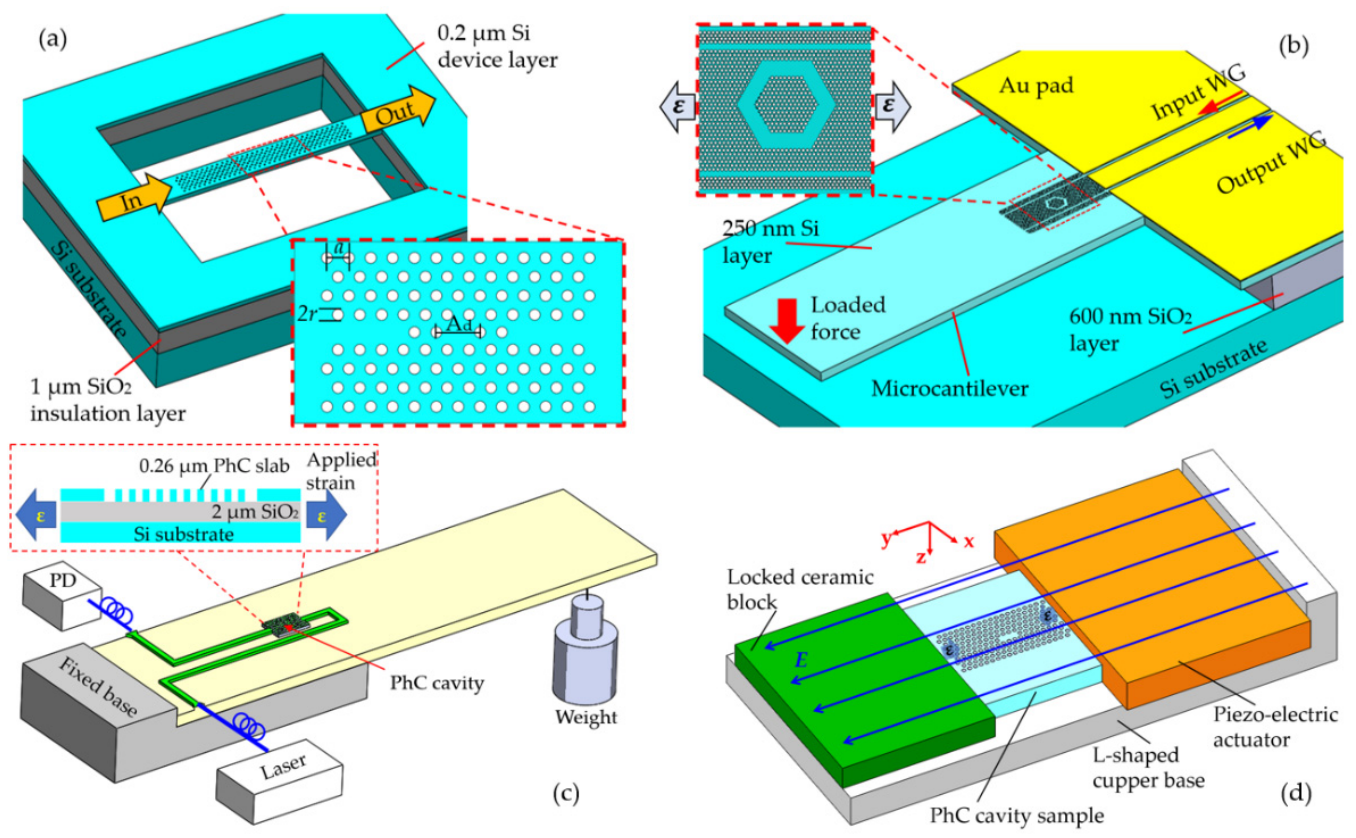

Figure 5. (a) Schematic of the pressure detection system based on PhC cavity (inset: layout of PhC using a hexagonal array of holes with the lattice constants $a, \mathrm{~A}_{d}$ and $r$ are the distance between the center two holes and radius of hole) [97]; (b) 3D illustration of 2D PhC slab strain sensors using PhC resonator structure (inset: deformed single ring PhC cavity driven by stress) [73]; (c) schematic of PhC cavity for strain sensing (inset: sectional view of strain applied on PhC) [98,99]; (d) schematic of electric field sensor with strain tuning of defect with PhC cavity [15].

Mechanically tunable 2D PhC cavities could also be achieved by varying the air-slot distance, which has been developed for displacement sensing. Yang et al. [9] theoretically demonstrated a micro-displacement sensor with a fixed and moving hole-array based slot PhC cavity, as shown in Figure 6a. The tuning of high-Q cavity was formed by laterally shifting two adjacent holes outwards in the opposite direction. It demonstrated a quasi-linear measurement of displacement with a sensitivity of $1.0 a^{-1}$ in simulation results. In a similar manner, Figure $6 \mathrm{~b}$ demonstrated a magnetometer based on an on-chip scale slot PhC cavity with movable and fixed sections [13]. An air-slot was inserted in a planar photonic crystal slab and high $\mathrm{Q}$ factors were attained by designing the dimensions of the air holes near the cavity center. As the movable mass of optical cavity assembly was affected by the magnetic field to displace the movable mass and change the width of air-slot, the alteration of the 
width of air-slot can be detect through the optical resonance. With an in-plane displacement sensitivity of $1.0 \mathrm{pm} / \mathrm{Hz}^{1 / 2}$, the enhanced magnetic sensitivity of $11.4 \mathrm{nT} / \mathrm{Hz}^{1 / 2}$ was achieved.
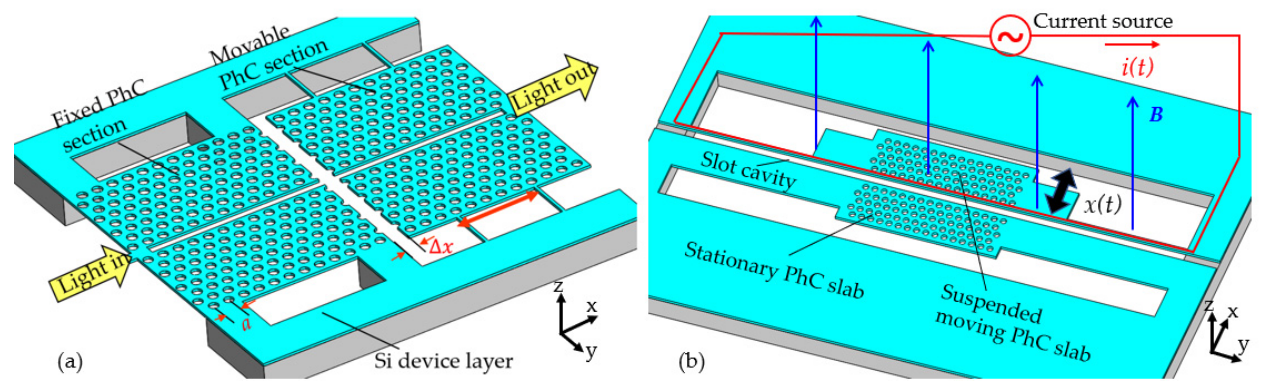

Figure 6. Schematics of the displacement sensors: (a) 2D-slot PhC with fixed and moving segments [9]; (b) schematic of the magnetic field sensor based on slot PhC slab cavity [13].

Out-of-plane deformation of 2D PhC cavity was also adopted for mechanical sensing applications. As shown in Figure $7 \mathrm{a}, \mathrm{a} \mathrm{Si}_{3} \mathrm{~N}_{4}$ cantilever was embedded in the center defect of 2D PhC cavity [77]. The bending of cantilever modulated the effective RI, and hence induced resonance shift of cavity. A theoretical minimum of surface stress of about $0.8 \mathrm{mN} / \mathrm{m}$ was solved by mechanical tuning the defect of $\mathrm{PhC}$ slab. Another analogous tunable $\mathrm{PhC}$ cavity integrated cantilever was proposed by directly inserting a cantilever into the middle of a PhC nanobeam [78], as illustrated in Figure $7 \mathrm{~b}$. The top $200 \mathrm{~nm}$ silicon layer served as an electrode while the thick silicon layer acted as a ground, and these two layers were electrically isolated by a $1-\mu \mathrm{m}$ silicon dioxide layer. The cantilever bent out of $\mathrm{PhC}$ slab and affected the band-edge resonance mode. Besides, a nonlinear behavior was observed in the impact of the cantilever's deflection on resonance shift. A maximum resonant wavelength shift of $13.5 \mathrm{~nm}$ was obtained under the applied voltage of $97 \mathrm{~V}$, however, the quality factor was relatively low because of the cavity loss caused by inserting the cantilever into the nanobeam cavity.
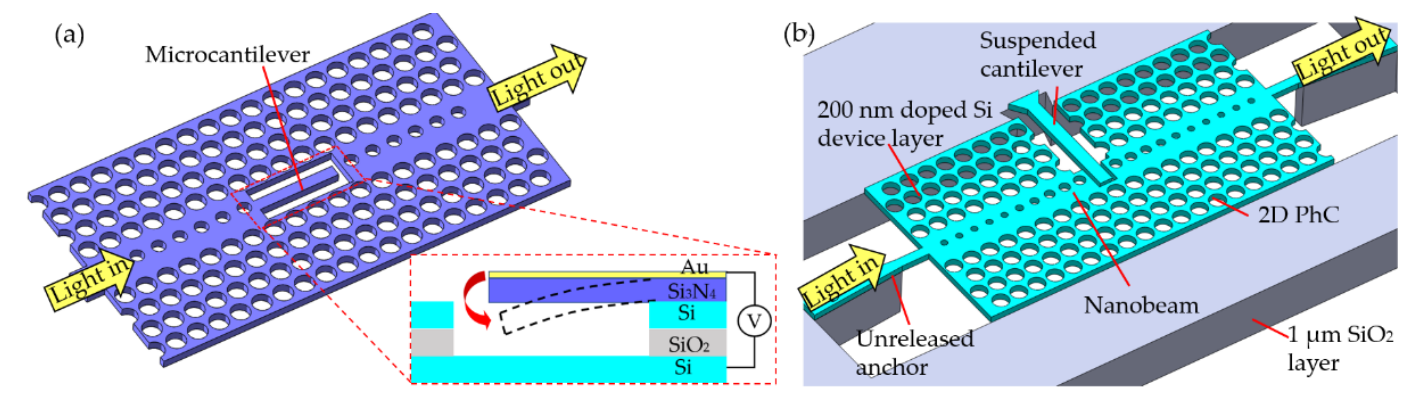

Figure 7. Schematics of the strain detection system based on cantilever-PhC cavity: (a) deflection of cantilever in the center of cavity (inset: the cross-section view for the electromechanical tuning) [77]; (b) cantilever inserted into a PhC nanobeam [78].

\subsubsection{Near-Field Probe Perturbation-Based PhC Sensors}

Resonance control of optical resonators can also be established using the evanescent field perturbation method. For 1D PCN cavities, their tuning configurations are illustrated in Figure 8a. An external nano-scale probe driven by a NEMS comb-drive actuator acted as a perturbation factor to the PCN cavity. To enhance the probe interaction with the evanescent optical field, Chew et al. $[8,42]$ reported a rectangular tip to tune a resonance shift of $1.1 \mathrm{~nm}$ with the decreasing gap between tip and cavity from 750 to $100 \mathrm{~nm}$. In comparison, a meniscus-like tip was used to tune the resonance shift of $2.25 \mathrm{~nm}$. However, tuning of a rectangular tip probe caused a significant drop of Q-factor and transmission of cavity, compared with a moderate degradation of Q-factor and transmission using the meniscus-like tip probe. The near-field perturbation tuning method was further developed with a nano-scale multi-tip probe [42] to reach a larger tuning range of $5.4 \mathrm{~nm}$. Besides $1 \mathrm{D} \mathrm{PhC}$ cavities, probe 
perturbation based 2D PhC cavities also has been developed for sensing applications. As shown in Figure $8 \mathrm{~b}$, a torsion-free pressure sensor was presented with the structure of a piston-rod side-coupled with the PhC membrane nanocavity [89]. The piston-rod was located on the center of PhC cavity and acted as a probe tip. It was attached to a silicon strip which can be deformed by applied strain. The stress displacement of silicon strip could change the depth of piston-rod inserted in the central nanohole of $\mathrm{PhC}$ nanocavity. A sensitivity reaching up to $0.50 \mathrm{~nm} / \mathrm{nN}$ was observed and its minimum detection force was estimated to be $0.68 \mathrm{nN}$. Furthermore, Yang et al. [100] proposed a three-dimensional force sensor. A shoulder-coupled $\mathrm{PhC}$ cavity was designed to detect forces in the horizontal and vertical directions, and a piston-rod $\mathrm{PhC}$ was used to detect the force in the upright direction. With a well-designed and optimized PhC structure, high sensitivities of $8.2 \mathrm{~nm} / \mu \mathrm{N}, 12.5 \mathrm{~nm} / \mu \mathrm{N}$, and $10.9 \mathrm{~nm} / \mu \mathrm{N}$ were obtained in the horizontal, vertical and upright directions, respectively; the detection limits for the three directions were $24 \mathrm{nN}, 16 \mathrm{nN}$, and $18 \mathrm{nN}$, respectively. However, the resonance tuning range obtained in the near-field probe perturbation was generally not very large, and additional energy losses that negatively affect the $Q$ factor and degraded optical transmission. Another sensing configuration based on the dielectric perturbation method in the PCN cavity sensors was present by Qiao et al. [101] for gas pressure measurement, as shown in Figure 9. In the PCN sensor, a polydimethylsiloxane (PDMS) membrane was coated on the functional silicon device area, which acted as a perturbation factor. As there was a pressure difference between the sealed holes and vacuum chamber, the deformation of PDMS could move close or away to the air holes of PCN cavity and then shift the resonant wavelength with the linear pressure sensitivity of $8.8 \mathrm{~nm} / \mathrm{MPa}$.

(a)

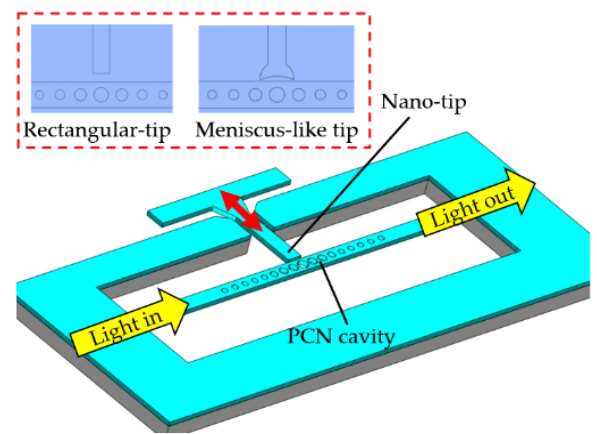

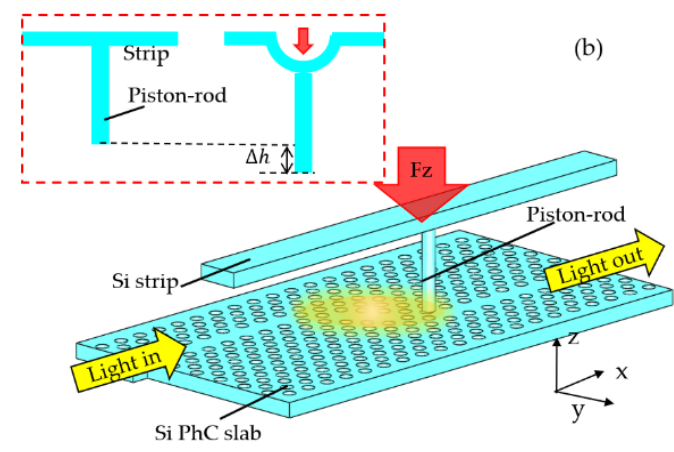

Figure 8. Schematics of the tunable PhC cavities based on near-field probe perturbation: (a) moveable probe-based single PCN cavity (inset: close-up view on two probe tips) [42]; (b) PhC side-coupled piston-rod microcavity (inset: depth variation $\Delta h$ due to the strain displacement) [89].
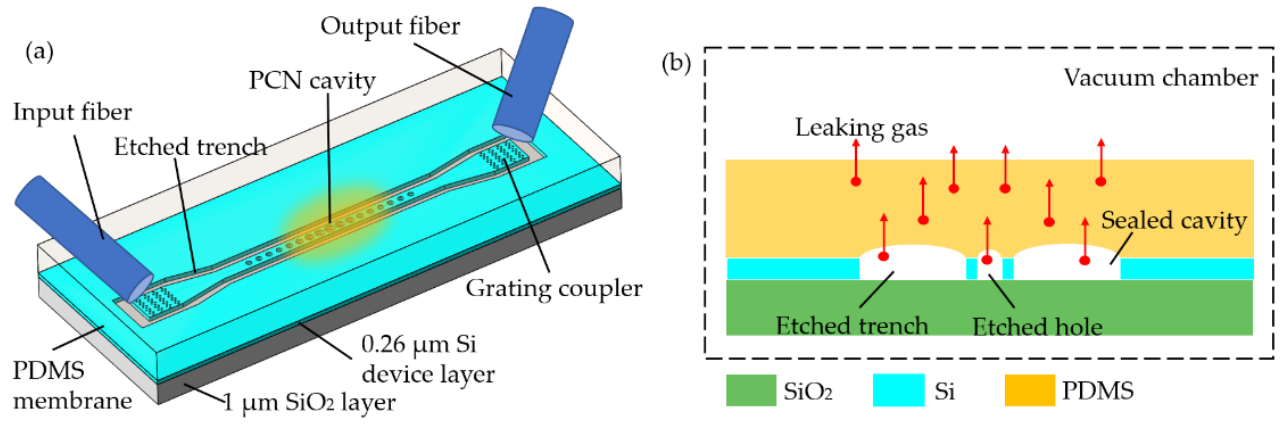

Figure 9. (a) Schematic of PCN cavity with polydimethylsiloxane (PDMS) coating; (b) cross-sectional view of gas cell and membrane deformation caused by pressure difference [101].

\subsubsection{Mode-Splitting Based Coupled PhC Sensors}

Mechanically tunable configuration can also be obtained via multi-coupled cavities by controlling the coupling strength between them. Taking a pair of PCN cavities coupled configuration in Figure 10a as an example, their coupling strength can be tuned by controlling the in-plane coupling gap to cause 
resonance shifts of even and odd modes. Such a configuration has attracted a lot of interests due to its potential for strong opto-mechanical interaction. Mechanically tunable coupled-PCN cavities could be modulated by the in-plane gap between two nanobeams (see Figure 10b) [49-52], in-plane laterally center offset (see Figure 10c) [53,54], out-of-plane rotation of suspended cavity (see Figure 10d) [55] as well as out-of-plane bending nanobeams (see Figure 10e) [56]. Du et al. [53] proposed a coupled PCN cavity by laterally driving a center-to-center offset to control the coupling strength dynamically, resulting in that optical mode splitting width decreased with the increment of lateral offset. Tian et al. [55] designed a torsion-induced coupled PCN cavity using a NEMS comb drive to demonstrate an optical spring effect with a maximum $77.1 \mathrm{~Hz}$ mechanical frequency drop by laser detuning. Besides, the out-of-plane coupled PCN cavity motion was presented using a vertical electrostatic force generated by tuning the potential difference between isolated layers [56]. With an applied voltage of $12 \mathrm{~V}$, an out-of-plane displacement could cause a redshift of $40.5 \mathrm{pm}$ in the second-order odd resonance mode and a blueshift of $63.5 \mathrm{pm}$ in the second-order even resonance mode.

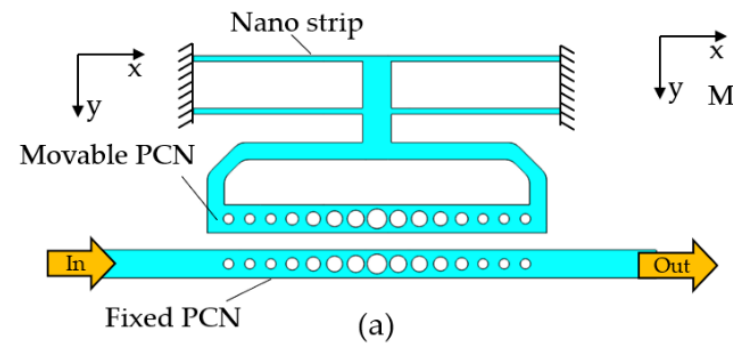

(a)

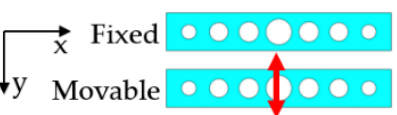

(b)

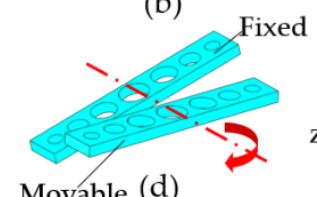

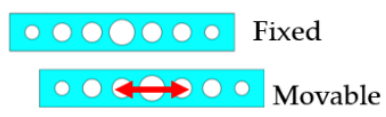

(c)

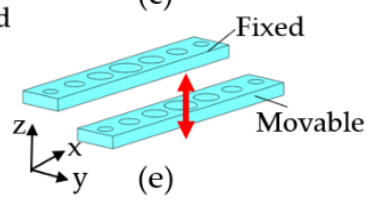

Figure 10. Schematics of mechanically tunable coupled PCN cavities: (a) doubly-coupled PCN cavity; (b) in-plane gap tuning [49-52]; (c) in-plane lateral center offset tuning [53,54]; (d) out-of-plane rotation [55]; (e) out-of-plane bending [56].

For sensing applications, a mechanically tunable coupled PCN cavity can also get its application in magnetic field sensing based on MEMS-actuated Lorentz force. As demonstrated in Figure 11a, a magnetometer was presented based on the resonance wavelength shift of a target supermode of the coupled PCN cavities. With the presence of magnetic field, the interaction between magnetic field and electric current in gold nano-wires could generate a Lorenz force to actuate a vertical offset variation between two coupled cavities. The resonance shift was caused by the Lorentz force-induced relative out-of-plane motion of movable nanobeam, thereby affecting the optical transmission [14]. The sensitivity and resolution were experimentally demonstrated as $22.9 \mathrm{mV} / \mathrm{T}$ and $48.1 \mu \mathrm{T} / \mathrm{Hz}^{1 / 2}$, respectively. Compared with other MEMS magnetometers, the proposed coupled PCN cavity sensor had a much smaller footprint. Thus, it could potentially achieve higher spatial sensing resolution of magnetic field. With an on-chip integrated NEMS actuator, another coupled PCN cavity was mechanically reconfigured in the lateral direction while keeping a constant separation gap [54], as shown in Figure 11b. By measuring the variation of resonance wavelength split width, the shearing optical force was detected at the scale sub-nano Newton $(\sim 0.29 \mathrm{nN})$. Such a lateral shearing optical force detection could be further studied in the applications of optomechanics and might be developed for optically driven tunable filters and optical switches.

Similarly, 2D PhC cavities can also be vertically coupled [79] by electrostatically actuating the out-of-motion controlled separation gap between two cavities. As a result, a resonant wavelength split can be obtained with the variation of vertical coupling gap. For sensing application, a numerical study on the double-layered PhC cavity was investigated under applied stress induced by an atomic force microscope (AFM) tip [12,102], as shown in Figure 12a. Two InGaAsP PhC microcavities were vertically coupled with a 400-nm air gap, and their mode properties and optical spectrum response were modulated when this gap varied with the structural bending of $\mathrm{PhC}$ cavity. The detectable stress limit was estimated to be as small as $0.95 \mathrm{nN}$ with an air gap of $165 \mathrm{~nm}$ in the presence of point AFM force. Experimentally, an on-chip displacement sensor was realized based on the 
double-membrane PhC slab integrated with electro-optical readout, as presented in Figure 12b [10,103]. The performance of the proposed displacement sensor was also characterized by nanomechanical actuation using a commercial AFM tip. It was demonstrated that the sensor had a sensitivity of $0.17 \mathrm{~nm} / \mathrm{nm}$ (wavelength/displacement) and a displacement noise floor of $7 \mathrm{fm} / \sqrt{\mathrm{Hz}}$.
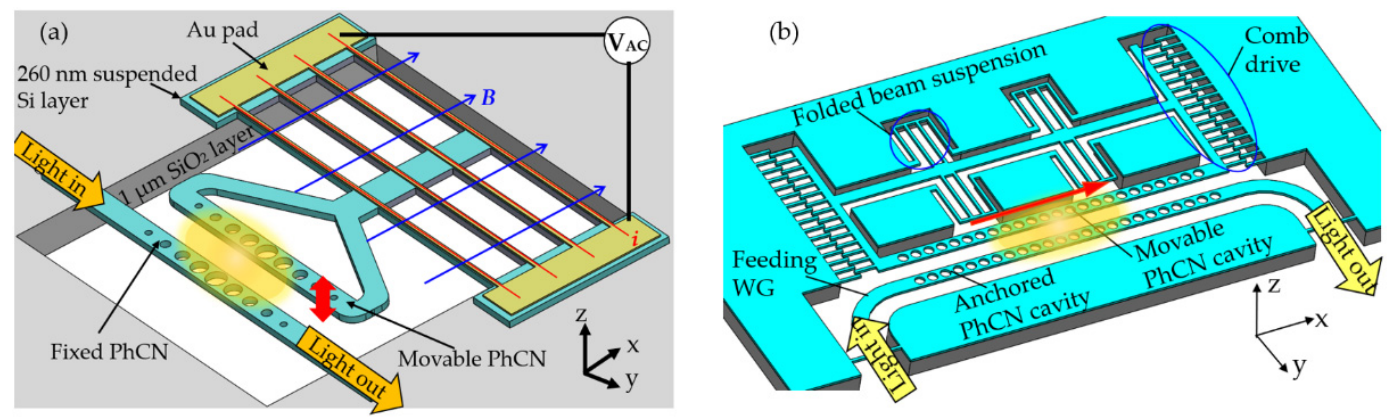

Figure 11. (a) Schematic of the magnetic field sensor based on coupled PCN cavity [14]; (b) schematic of coupled PCN cavity to detect lateral shearing optical force [14].
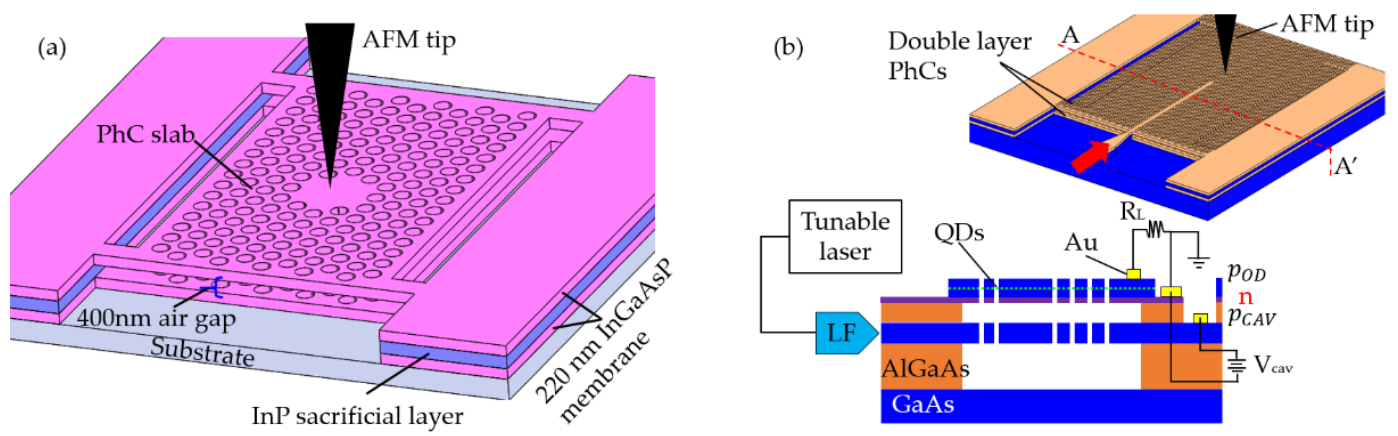

Figure 12. Schematics of the strain sensors: (a) double-layer PhC cavity using an atomic force microscope (AFM) tip contacting the center of microcavity to apply strain [12,102]; (b) displacement sensor using a double-membrane $\mathrm{PhC}$ slab integrated with electro-optical readout $[10,103]$.

\subsection{Discussion}

As discussed above, there are mainly three working mechanisms used for mechanically tunable $\mathrm{PhC}$ cavity sensing applications, including probe perturbation, deformable cavity as well as mode splitting methods. Table 3 summarizes a comparison of mechanically tunable $\mathrm{PhC}$ with various photonic configurations, sensing principles, measurable factors, remarks and sensing applications. Most sensing applications based on mechanically tunable $\mathrm{PhC}$ cavities essentially utilize the principle of displacement sensing. The target physical signals, such as force, stress, magnetic field, etc., are converted to the displacement of an opto-mechanical sensor. In order to optimize the sensing performance, the optimized mechanical design is required for efficient conversion from the target signal to on-chip displacement. Besides, simultaneous optimization on the optical and mechanical components in PhC cavities should be well-designed with a higher opto-mechanical coupling efficiency to maximize the variation of optical readout. However, its sensitivity is still limited by thermal-electronic noise [104] in the readout circuits and the operational bandwidth only reaches up to a level of $\mathrm{kHz}$. Considering sensitivity and bandwidth, another opto-mechanical system driven by radiation force is expected to behave well and will be discussed in next section. In the future, with further integration with PICs, mechanically tunable micro/nano PhC cavities are expected to evolve with compact and feasible measurement apparatus and find more promising applications in optical communications. 
Table 3. Comparison of sensing applications based on mechanically tunable $\mathrm{PhC}$ cavities.

\begin{tabular}{|c|c|c|c|c|c|c|}
\hline \multicolumn{2}{|c|}{ PhC Cavity } & Principle & $\begin{array}{l}\text { Tunable } \\
\text { Movement }\end{array}$ & Factor & Remarks & $\begin{array}{c}\text { Sensing } \\
\text { Applications }\end{array}$ \\
\hline \multirow{5}{*}{ Single $\mathrm{PhC}$} & PCN & Probe perturbation & In-plane translating & $\begin{array}{c}\lambda^{*} \\
\text { and } Q^{+}\end{array}$ & $\begin{array}{l}\text { Small wavelength tuning range; } \\
\text { Moderate optical loss }\end{array}$ & Displacement $[8,42]$; \\
\hline & \multirow{3}{*}{$\begin{array}{l}\text { 2D PhC } \\
\text { Slab }\end{array}$} & Probe perturbation & Vertical translating & $\lambda$ & $\begin{array}{l}\text { Large wavelength tuning range small } \\
\text { optical loss }\end{array}$ & $\begin{array}{l}\text { Displacement [89]; } \\
\text { Force [100] }\end{array}$ \\
\hline & & Deformable cavity & In-plane translating & $\lambda$ & Large wavelength tuning range & $\begin{array}{l}\text { Displacement [9]; } \\
\text { Magnetic field [13] }\end{array}$ \\
\hline & & Deformable cavity & N/A & $\lambda$ & Small wavelength tuning range & $\begin{array}{l}\text { Strain }[11,98,99] \\
\text { Electric field [15] }\end{array}$ \\
\hline & Cantilever-PhC slab & Deformable cavity & Out-of-plane bending & $\lambda$ and $Q$ & Large optical loss & Stress [77] \\
\hline \multirow[t]{2}{*}{ Coupled PhCs } & $\begin{array}{l}\text { Coupled } \\
\text { PCN }\end{array}$ & Mode splitting & $\begin{array}{l}\text { In-plane translating } \\
\text { Out-of-plane } \\
\text { rotating/bending }\end{array}$ & $\lambda$ & $\begin{array}{l}\text { Moderate wavelength tuning range; } \\
\text { High operation speed and resolution }\end{array}$ & $\begin{array}{l}\text { Magnetic field [14]; } \\
\text { Force [54] }\end{array}$ \\
\hline & $\begin{array}{l}\text { Vertically Coupled } \\
\text { PhC }\end{array}$ & Mode splitting & Vertical translating & $\lambda$ and $Q$ & $\begin{array}{l}\text { Large wavelength tuning range with } \\
\text { high Q-factor; } \\
\text { High sensitivity }\end{array}$ & $\begin{array}{l}\text { Stress [12]; } \\
\text { Displacement [10]; }\end{array}$ \\
\hline \multirow[t]{2}{*}{ Split PCN } & Longitudinally split & Deformable cavity & $\begin{array}{l}\text { In-plane translating } \\
\text { Out-of-plane bending }\end{array}$ & $\lambda$ and $Q$ & Large Q-factor tuning range & $\begin{array}{l}\text { Displacement } \\
\text { [65-69] }\end{array}$ \\
\hline & Transversely split & Deformable cavity & In-plane translating & $\lambda$ and $Q$ & Wide tuning wavelength range & $\begin{array}{l}\text { Rotation and } \\
\text { displacement [96] }\end{array}$ \\
\hline
\end{tabular}

${ }^{*} \lambda$ indicates monitoring the resonance shift; ${ }^{\dagger} \mathrm{Q}$ indicates monitoring $\mathrm{Q}$-factor variation. 


\section{Radiation Pressure-Driven PhC Cavity Sensors}

In addition to the mechanically tunable PhC cavities, sensing applications can also be realized using radiation pressure-driven $\mathrm{PhC}$ cavities. Along with the high-Q optical mode in a PhC cavity, the mechanical mode can be excited at the same time. The optical and mechanical modes are efficiently coupled to each other, resulting in strong optomechanical coupling. A radiation pressure rises from the optomechanical coupling in the interaction of optical and mechanical cavities. The essential effect of the optomechanical coupling is the fact that the optical force induced by the mechanical displacement of cavity modulates the state of light. In this section, sensing applications based on PhC cavity optomechanics for high precision detection of displacement, mass, force, acceleration, etc. are reviewed.

\subsection{Optomehanical Effects for Sensing Applications}

The starting point of subsequent radiation pressure-driven opto-mechanical interaction is briefly referred to cavity optomechanics. Radiation pressures, also known as optical forces, become obvious in the presence of strong gradients of optical mode field confined within nanomechanical holes or slots [17]. Here we take a model based on F-P cavity to discuss optomechanical phenomena, as illustrated in Figure 13a. In the optomechanical system, light with frequency $\omega_{\text {cav }}$ is confined inside a high Q-factor $Q_{o p t}$ optical cavity. Meanwhile, one of the mirrors of optical cavity with the effective mass $m_{e f f}$ is coupled with a mechanical oscillator represented with a spring constant $k=2 m_{e f f} \Omega_{m}^{2}$. The mechanical oscillator with its mechanical quality factor $Q_{m}$ vibrates at its mechanical frequency $\Omega_{m}$. The motion $x$ of mechanical oscillator brings about a variation in the optical path length to alter cavity resonance $\omega_{c a v}$, which in turn causes a change of light amplitude, thereby varying the radiation pressure acting on the motion to close this optomechanical backaction. We refer to this feedback-loop backaction as dynamic backaction, as presented in Figure 13b. In this feedback-loop, it starts with a mechanical oscillator subject to an input force $d F$ (thermal forces or applied signal forces) to drive a mechanical movement $x$. Due to the optomechanical coupling $g_{o m}$, mechanical movement tunes optical cavity field $d \alpha$, wherein $d \alpha_{o u t}$ contains information on the mechanical position. Besides, the amplitude fluctuation $d \alpha$ is also coupled back to the mechanical oscillator via laser detuning, resulting in a backaction. Finally, the light amplitude change-induced radiation pressure takes effect on the mechanical oscillator, closing the feedback loop.
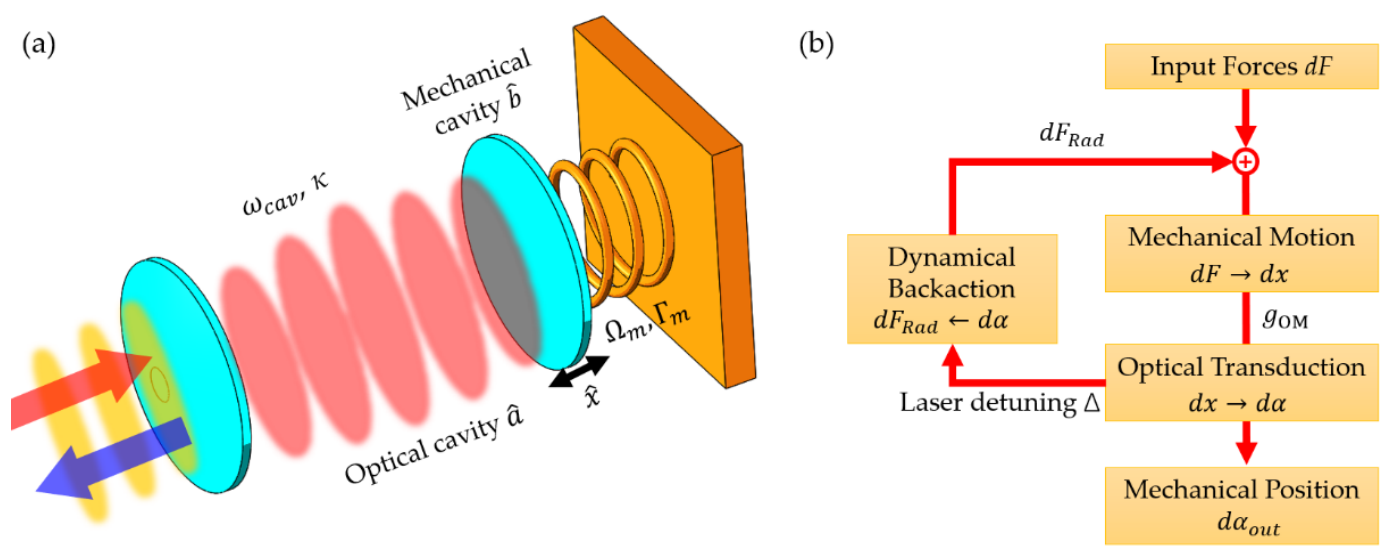

Figure 13. (a) Canonical schematic of a cavity optomechanical system with a laser-driven optical cavity and a vibrating mechanical cavity; (b) schematic of optomechanical backaction loop [17].

As described above, this dynamic backaction in optomechanical system can be represented by the coupled optical and mechanical resonator by an interaction Hamiltonian function as $H_{\text {int }}=$ $\hbar g_{o m} x \hat{a}=\hbar g_{0} \hat{a} \hat{a}(\hat{b}+\hat{b})$, where $g_{0}=g_{o m} x_{Z P F},\left(x_{Z P F}=\sqrt{\hbar / 2 m_{e f f} \Omega_{m}}\right.$ is the zero-point fluctuation 
and $\left.g_{o m}=\partial \omega_{c a v} / \partial x\right)$, denotes the single photon coupling strength; $\hat{a}$ and $\hat{b}$ denote photon and phonon annihilation operators with $\hat{a} \hat{a}$ the circulating photon number. The cavity optomechanics induced radiation pressure can be given by the derivation $\hat{H}_{\text {int }}$ to mechanical motion $x$, given as $F_{\text {opt }}=-\frac{\partial \hat{H}_{\text {int }}}{\partial x}=-\hbar g_{o m} \hat{a} \hat{a}$. In optomechanical sensing applications, this optical force can be used to develop optomechanical sensors with intrinsic advantages over traditional mechanically tunable methods on bandwidth, speed and efficiency [105]. Considering the effect of $F_{\text {opt }}$, the optomechanical interaction equation to indicate coupled light amplitude with mechanical position is given as,

$$
\left\{\begin{array}{c}
\dot{\alpha}=-\frac{\kappa}{2} \alpha+i(\Delta+G x) \alpha+\sqrt{\kappa_{e x}} \alpha_{i n} \\
m_{e f f} \ddot{x}=-m_{e f f} \Omega_{m}^{2} x-m_{e f f} \Gamma_{m} \dot{x}+\hbar G|\alpha|^{2}
\end{array}\right.
$$

where $\alpha$ and $\alpha_{i n}$ are the classical correspondence of cavity mode $a$ and input field mode $a_{i n}$, respectively; $\kappa$ and $\kappa_{e x}$ refer to the total and external decay rates of cavity mode; $\Delta=\omega_{L}-\omega_{c a v}$ represents laser detuning between laser and optical cavity. In the presence of optomechanical interaction, the dynamical backaction can be deduced from the change of mechanical susceptibility as

$$
\sum(\omega)=\chi_{m, m_{e f f}}^{-1}-\chi_{m}^{-1}=2 m_{e f f} \Omega_{m} g^{2}\left[\frac{1}{(\Delta+\omega)+i \kappa / 2}+\frac{1}{(\Delta-\omega)-i \kappa / 2}\right]
$$

To observe dynamical behaviors arising from optomechanical coupling, the real and imaginary parts of Equation (6) can yield the mechanical eigenfrequency shift $\delta \Omega_{m}(\omega)$ and optical damping rate $\Gamma_{\text {opt }}(\omega)$ in the strong optomechanical coupling regime $\left(g>\kappa / 2\right.$ and $\kappa \ll \Omega_{m}$, i.e., resolved sideband) as

$$
\begin{aligned}
& \delta \Omega_{m}(\omega)=g^{2} \frac{\Omega_{m}}{\omega}\left[\frac{\Delta+\omega}{(\Delta+\omega)^{2}+\kappa^{2} / 4}+\frac{\Delta-\omega}{(\Delta-\omega)^{2}+\kappa^{2} / 4}\right] \\
& \Gamma_{\text {opt }}(\omega)=g^{2} \frac{\Omega_{m}}{\omega}\left[\frac{\kappa}{(\Delta+\omega)^{2}+\kappa^{2} / 4}+\frac{\kappa}{(\Delta-\omega)^{2}+\kappa^{2} / 4}\right]
\end{aligned}
$$

Equation (7) indicates optical spring effect, where shift of mechanical frequency can be deemed to modify mechanical stiffness as $\Omega_{m}=\sqrt{k / m_{e f f}}$. Since $\Gamma_{\text {opt }}$ in Equation (8) causes the change in total effective mechanical damping as $\Gamma_{e f f}=\Gamma_{m}+\Gamma_{\text {opt }}$, extra optical damping leads to cooling while anti-damping can lead to amplification. Both optical spring and damping behaviors can be observed in experiments under different ratios of $\Omega_{m} / \kappa$ [106]. In Doppler regime $\left(\kappa \gg \Omega_{m}\right)$, mechanical frequency shift caused by the pump light yields $\left.\delta \Omega_{m}\left(\Delta=\omega_{L}-\omega_{\text {cav }}\right)\right|_{\kappa \gg \Omega_{m}}=g^{2} \frac{2 \Delta}{\Delta^{2}+\kappa^{2} / 4}$. It implies that the spring of mechanical oscillator will be softened under the red laser detuning $(\Delta<0)$ and hardened under the blue laser detuning $(\Delta>0)$. However, mechanical frequency shift becomes remarkably different when it works in the resolved sideband regime $\left(\Omega_{m} \gg \kappa\right)$, where optical spring effect vanishes at certain laser detuning and radiation pressure contributes only to cooling or amplification function [107].

When it comes to sensing applications, excellent sensing performance can be achieved through the optical readout of mechanical characteristics from optomechanical system. Aim to obtain the maximum mechanical signal readout, the probing laser wavelength should be placed at the point of the highest slope of optical resonance, as depicted in Figure 14a [108,109]. As light cycles inside the cavity, a radiation pressure induced by optomechanical coupling can interplay with mechanical resonator. Under the blue-detuned $\left(\Delta_{L}=\lambda_{L}-\lambda_{c a v}<0\right)$ laser wavelength, the motion of mechanical resonator is strengthened due to optomechanical interaction. This dynamic backaction can result in the optical spring effect, namely, setting of laser detuning $\Delta_{L}$ determines the behavior of mechanical frequency $\Omega_{m}$. The concept of optomechanical spring sensing is represented in Figure 14b,c where a high optical Q-factor cavity is employed. Any tiny perturbation induced by nanoparticle adhesion or physical motion causing optical resonance shift $\delta \lambda$ could be converted to the mechanical frequency shift as 
$\delta \Omega_{m}=-\frac{d \Omega_{m}}{d \Delta_{L}} \delta \lambda$. Hence, it provides a valid optomechanical sensing mechanism through amplifying the optical resonant wavelength. The minimal detectable wavelength shift of this sensing scheme is determined by its mechanical linewidth $\Delta \Omega_{m}$ as,

$$
\left(\frac{\delta \lambda}{\lambda_{\text {cav }}}\right)_{\min }=\frac{\Delta \Omega_{m} / \lambda_{\text {cav }}}{-d \Omega_{m} / d \Delta_{L}}
$$
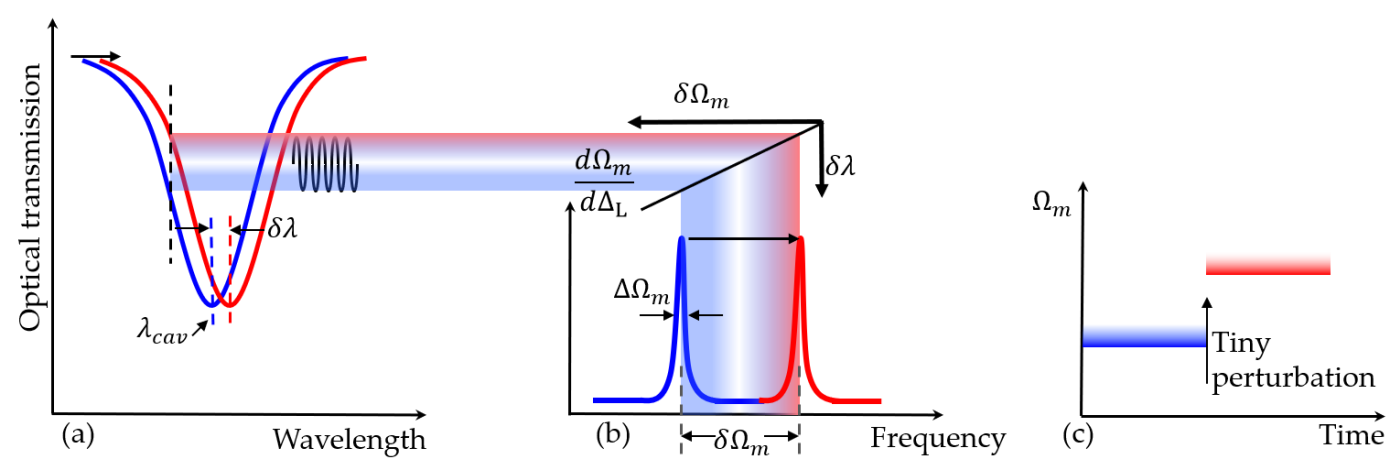

Figure 14. Schematic illustrating optical spring sensing mechanism [108]: (a) optical transmission spectrum; $(\mathbf{b}, \mathbf{c})$ mechanical spectra shift due to the applied tiny perturbation.

Obviously, it can be seen from Equation (9) that a narrow mechanical linewidth $\Delta \Omega_{m}$ and a large frequency tuning rate $d \Omega_{m} / d \Delta_{L}$ arising from optical spring effect could provide an excellent approach to detect the optical resonance variation. By defining the sensing resolution as $\left(\delta \lambda / \lambda_{\text {cav }}\right)_{\min }=\left(\eta_{\text {om }} Q_{m}^{e f f} Q_{\text {opt }}\right)^{-1}$, where $Q_{m}^{e f f}=\Omega_{m} / \Delta \Omega_{m}$ is the effective mechanical Q-factor and $\eta_{o m}$ denotes optomechanical transduction efficiency depending on laser detuning $\Delta_{L}$, it can be seen that the sensing resolution depends not only on $Q_{o p t}$ of optical cavity in conventional microcavity sensors, but also on the mechanical $Q_{m}^{e f f}$ of optomechanical cavity. Compared with conventional mechanical tunable sensing methods, optomechanical sensing can enhance the sensing resolution by about a mechanical quality factor of $Q_{m}^{e f f}$.

\subsection{Sensing Applications Based on Radiation Pressure}

\subsubsection{Displacement Sensing}

Highly sensitive displacement detection lays the technical foundation for other sensing applications because variations of other physical parameters can be converted to the movement of an on-chip component. Optomechanical photonic cavities are excellent for displacement sensing due to the strong interaction between the mechanical resonator and photonic cavity. However, displacement sensing based on optical cavities is technically limited by fundamental quantum shot noise on the photodetector [110]. The level of quantum shot noise decreases as the square-root of input light power. As the optical intensity further increases, the shot noise arising from optical cavity can lead to an oscillating optical force on the mechanical object, eventually causing the fluctuation of displacement. In the position measurement, standard quantum limit (SQL) provides a comparison scale to evaluate whether the measurement imprecision of displacement is sufficient to distinguish the zero-point fluctuation $(\mathrm{ZPF})$ of mechanical resonator At the SQL, the backaction noise $S_{x x}^{S Q L}$ induced by optical force is equal to the imprecision shot noise $S_{x x}^{Z P F}$ caused by displacement fluctuation $[20,111]$. In this critical condition, SQL is equal to the ZPF of mechanical motion given as $x_{S Q L}=\sqrt{\hbar / 2 m \Omega_{m}}$. At the resonance, SQL is also defined by $S_{x x}^{S Q L}=\hbar /\left(m \Omega_{m} \Gamma_{m}\right)$, which corresponds to a quarter of mechanical quantum noise energy.

To overcome this limit, displacement detection below SQL can theoretically be achieved by applying squeezed light for input laser, and this technological breakthrough has been experimentally 
demonstrated in a gravitational wave measurement [112]. In the zipper-cavity experiment shown in Figure 15a, the in-plane differential mechanical motion induced by radiation-pressure, modifying the gap between nanobeams, strongly modulated optical frequency with an optomechanical coupling rate of $g_{0} / 2 \pi=1 \mathrm{MHz}$. A level of $4.5 \pm 0.5 \%$ squeezing with a bandwidth of a few $\mathrm{MHz}$ at the mechanical frequency of $\Omega_{m}=28 \mathrm{MHz}$ was experimentally obtained. Radiation-pressure backaction fluctuation was greatly inhibited and affected by the motion of mechanical resonator, which demonstrated a fundamentally quantum aspect of displacement measurement. Another adiabatic tapered waveguide-PhC cavity scheme was developed into an on-chip optomechanical device [61], as shown in Figure 15b. Note that a mounted fiber tip well-aligned with a coupled waveguide could realize a coupling efficiency of $52 \%$ at the resonance wavelength. The measured imprecision noise floor at the fundamental in-plane mechanical mode of $3.3 \mathrm{MHz}$ revealed a factor of 2.8 above SQL for continuous displacement sensing. The mechanical resonator with an effective mass of $15 \mathrm{pg}$ was equal to a zero-point amplitude reaching up to $x_{Z P F}=13 \mathrm{fm}$. In addition, several technical conditions of high Q-factor and mechanical frequency, as well as cryogenic operation $(10 \mathrm{~K})$, were essential to restrain the effect of thermal noise. Future improvements could exploit alternative photonic material platforms for higher Q-factors, such as diamond [113,114] or silicon carbide $[115,116]$.
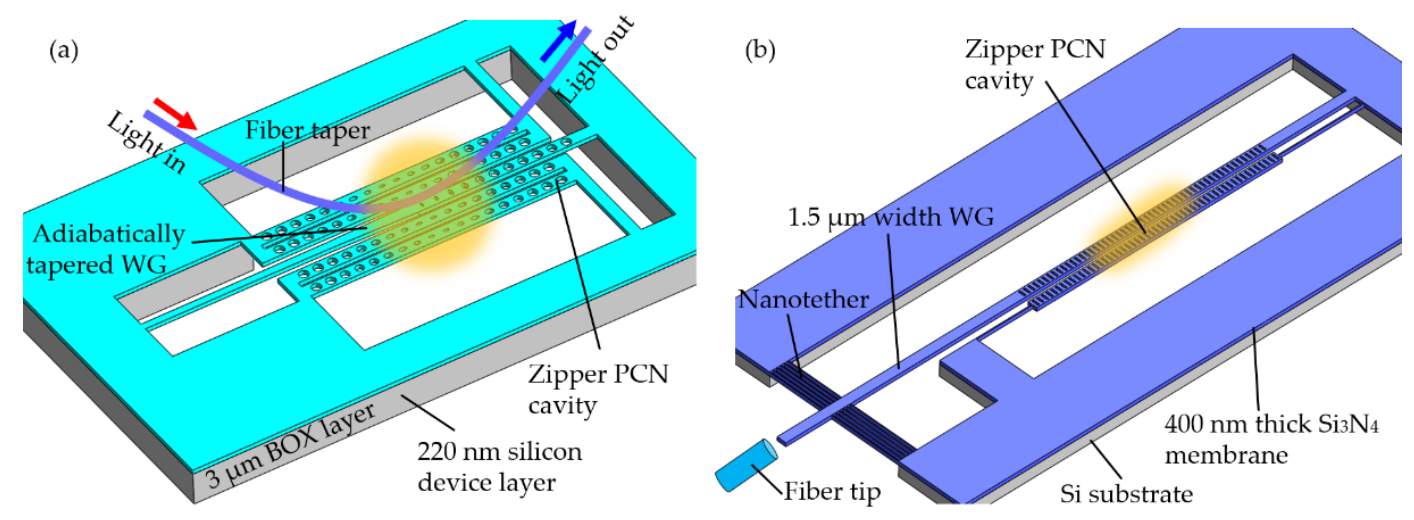

Figure 15. Schematics of squeezed light in optomechanics for displacement sensing: (a) waveguidecoupled zipper optomechanical PCN cavity [112]; (b) optical fiber tip routed zipper PCN cavity [61].

Nonlinear optomechanics occurs when the optical cavity frequency couples into the square of mechanical displacement in the radiation pressure interaction, which have been exploited in the realization of motion measurements. The first optomechanical PhC cavity system was featured with strong nonlinear optomechanical coupling, low mass as well as large optical mode displacing, as illustrated in Figure 16. A suspended paddle-like nanocavity [117-119] with hundreds of femtograms of mass could support several mechanical resonances (see inset in Figure 16) driven by the radiation pressure at the cavity resonance. This paddle nanocavity was nonlinearly coupled to a PhC nanocavity with a quadratic optomechanical coupling coefficient $g_{2}>2 \pi \times 400 \mathrm{MHz} / \mathrm{nm}^{2}$ and an optomechanical coupling rate of $\Delta \omega_{0}>2 \pi \times 16 \mathrm{~Hz}$. Additionally, thermally driven mechanical movements of the paddle nanocavity were also coupled and translated by observing resonances in the mechanical spectrum. The paddle nanocavity was useful for optomechanical force sensing and nonlinear optomechanics. To enhance the performance of the square of mechanical motion, a large position-squared optomechanical coupling was realized in a planar silicon PhC cavity [120], as presented in Figure 17a. By tuning the coupling rate of mode splitting in PhC cavities, it experimentally demonstrated a position-squared vacuum coupling rate reached up to $\mathrm{g} / 2 \pi=245 \mathrm{~Hz}$ inferred between the optical supermodes. Under the action of radiation pressure, it excited the fundamental in-plane mechanical motion at the frequency of $\Omega_{m} / 2 \pi=8.7 \mathrm{MHz}$, which corresponded to a coupling displacement coefficient of $\mathrm{g}^{\prime} / 2 \pi=1 \mathrm{THz} / \mathrm{nm}^{2}$. In comparison to other photonic schemes, due to the strong optomechanics effect and flexible mechanical structure, the position-squared coupling rate was much larger than that reported in the microdisk-cantilever [121] and paddle nanocavity [117] systems. 
Another displacement transducer with an imprecision below SQL was demonstrated using a sliced PCN cavity, as shown in Figure 17b [47]. Light in the sliced PCN resonator was highly confined in the nanoscale volume to create a radiation pressure to move the interface of sliced cavity with a low mass of $\sim 2.4 \mathrm{pg}$, which lead to unprecedented optomechanical interaction strength of $\mathrm{g} / 2 \pi \approx 11.5 \mathrm{MHz}$. The nonlinear transducer with a relatively large cavity bandwidth $(0.5 \mathrm{THz})$ is attractive for system integration and miniature sensor technologies in nano-optomechanical systems.

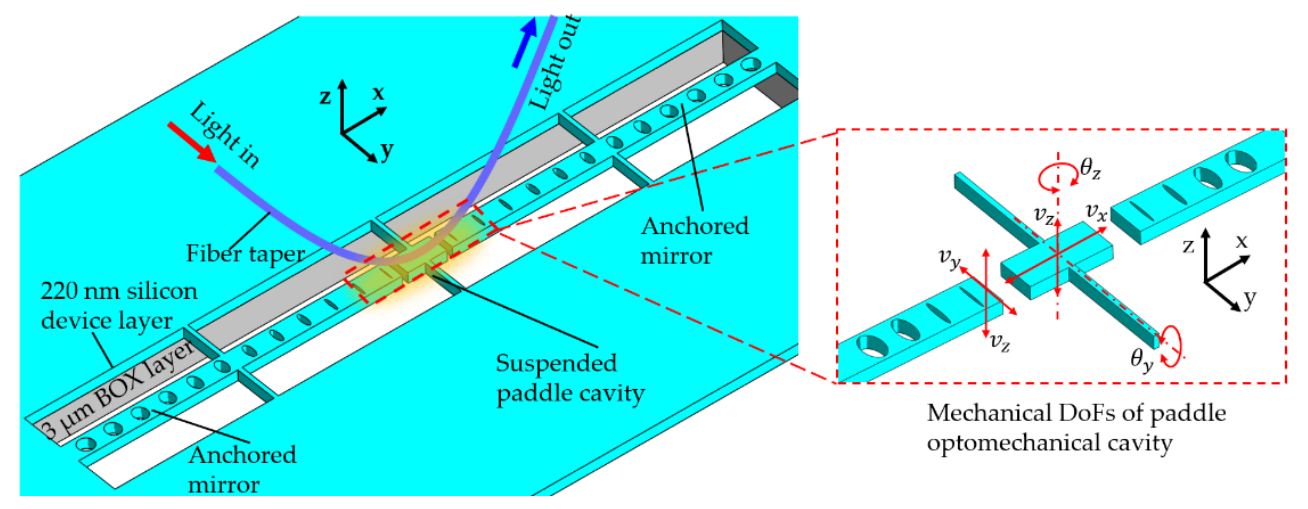

Figure 16. Schematics of photonic crystal paddle nanocavity (inset: mechanical modes of paddle cavity) [117-119].
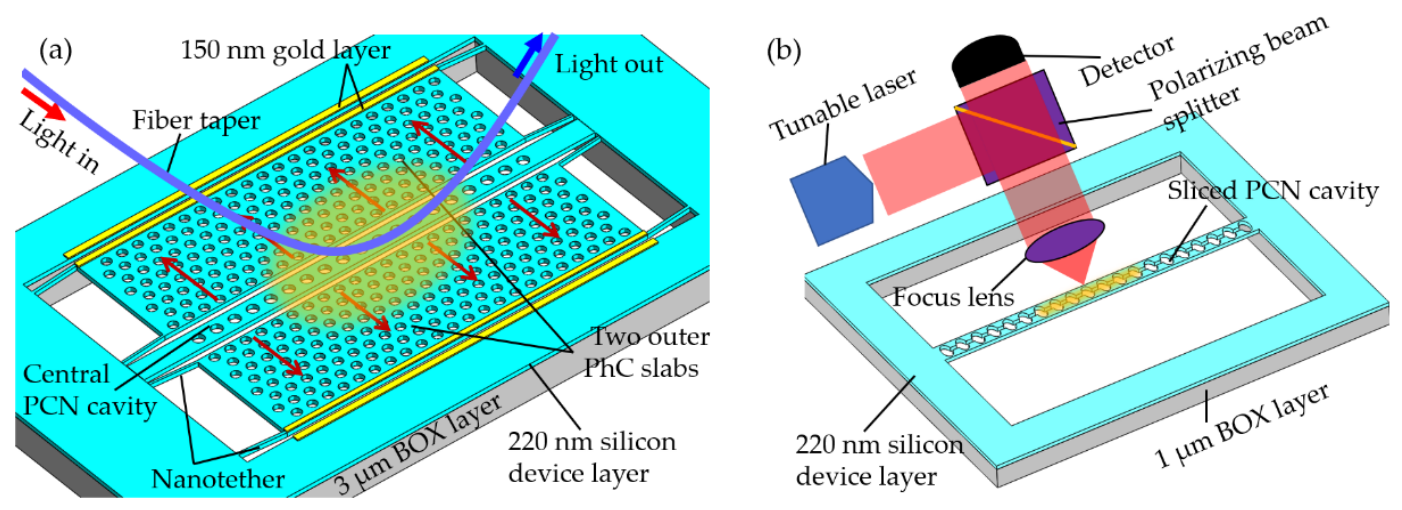

Figure 17. Schematics of optomechanical crystals for displacement sensing: (a) a central PCN cavity coupled to double-slotted photonic crystal slabs [120]; (b) sliced PCN cavity [47].

$\mathrm{PhC}$ cavities are significantly promising to provide the platform for on-chip integration with MEMS technologies, wherein large radiation pressure can be excited due to the strong optomechanical coupling in the nanoscale mode volume. Therefore, a chip-scale platform for studying the mechanical degree of freedom induced by radiation pressure is achieved by integrating cavity optomechanics with MEMS, where optomechanical system demonstrates near quantum-limited motion read-out and strong radiation pressure backaction. As shown in Figure 18, the electromechanical control of cavity air slot width could tune the optical cavity resonance in the slot 2D PhC membrane cavity $[82,122]$. The strong light confinement in the air slot region made the optical mode frequency ultrasensitive to the separation of two PhC membranes. The optical field was localized inside the air-slot nanocavity between two PhC membranes, which gave rise to the radiation pressure due to strong optomechanical coupling. It was demonstrated that a wide-band $(\sim 19 \mathrm{~nm})$ electromechanical tuning of the optical resonance near shot-noise limited optical read-out of mechanical motion at the acoustic mode frequency of 3.6 MHz [82]. Likewise, another all-integrated MEMS was developed in an ultrahigh Q-factor slotted PhC microcavity [123]. Both electromechanical and optomechanical interactions yield effective electrical actuation and sensitive optical transduction of mechanical modes of the PhC membrane. The sixth order mechanical mode was identified with a high frequency reaching up to $4.2 \mathrm{GHz}$ 
with the clamping limited mechanical Q-factors ranging from 240 to 1730. Electro-optomechanical systems have the merits of wide-range tuning, fast electrical control of optical resonances, and electrically tunable radiation pressure backaction effects. Such hybrid systems could be applied to achieve the signal conversion between optical and microwave frequency domains and are expected to exploit high-performance sensing applications for radiation pressure enforced displacement, mass and magnetometry measurements.

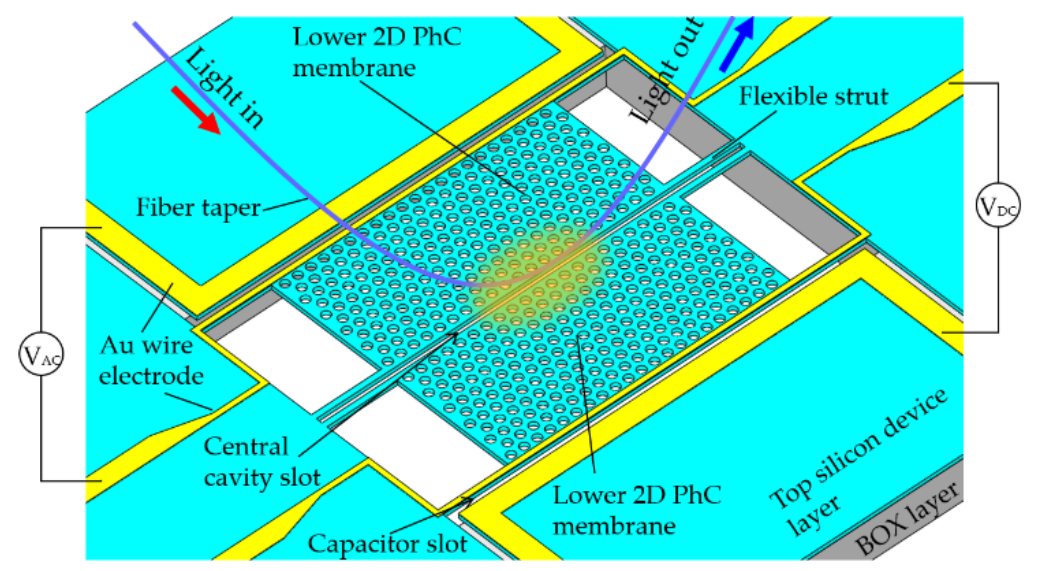

Figure 18. A tunable slotted-waveguide PhC electro-optomechanical cavity [82,122].

\subsubsection{Force Sensing}

Displacement of the mechanical resonator is closely related with applied force by the mechanical susceptibility $\chi_{x x}$ as defined in Fourier transformation of $x(\omega)=\chi_{x x}(\omega)\left[F_{e x}(\omega)+F_{L}(\omega)\right]$, where $F_{e x}(\omega)$ and $F_{L}(\omega)$ are applied external force and noise force. A combination of large optomechanical coupling $g_{o m}$, large mechanical susceptibility (low mass $m_{e f f}$ and high $Q_{m}$ ) and sharp optical resonance (large $\left.Q_{\text {opt }}\right)$ contributes to sensitivity-enhanced radiation pressure-driven devices. Miniature mechanical structures on the sub- $\mu \mathrm{m}$ scale with high mechanical frequency and low stiffness are highly preferred on account of their good immunity to environmental vibration and sensitivity. Therefore, PCN cavities are more attractive in these sensing applications due to their ultralow mass, flexural mechanics and high optical Q-factors. Currently, force sensors based on PhC cavities are widely studied [124-126]. As shown in Figure 19a, a split PCN cavity had two cantilever nanomechanical resonators serving as optical mirrors [124]. Mechanical resonances of the suspended mirror could be efficiently excited by several sources (indicated by red arrows in Figure 19a). High optomechanical coupling in the nanocavities was realized when mechanical motion of suspended mirror controlled the splitting gap. As the nanocavity length effectively modified, resulting in a dispersive coupling to optical resonance. Mechanical resonances of the suspended mirror were optically coupled through radiation pressure, thereby allowing sensitive torque measurements. It was demonstrated that $500 \mathrm{MHz} / \mathrm{nm}$ dissipative coupling rate and $2 \mathrm{GHz} / \mathrm{nm}$ dispersive coupling rate were capable to detect mechanical resonances with the torque sensitivity of $1.2 \times 10^{-20} \mathrm{Nm} / \sqrt{\mathrm{Hz}}$ in ambient condition and $1.3 \times 10^{-21} \mathrm{Nm} / \sqrt{\mathrm{Hz}}$ in low vacuum. Likewise, a suspended paddle-like nanocavity [126] in the middle of the split PCN cavity modified the gap between two optical mirrors due to the torsional and feasible mechanical mode of paddle cavity, as shown in Figure 19b. The floating motions of suspended paddle nanocavity were supported by two nanotethers, and the mechanical resonances excited by applied torsion could be modulated and detected through optomechanical coupling. 

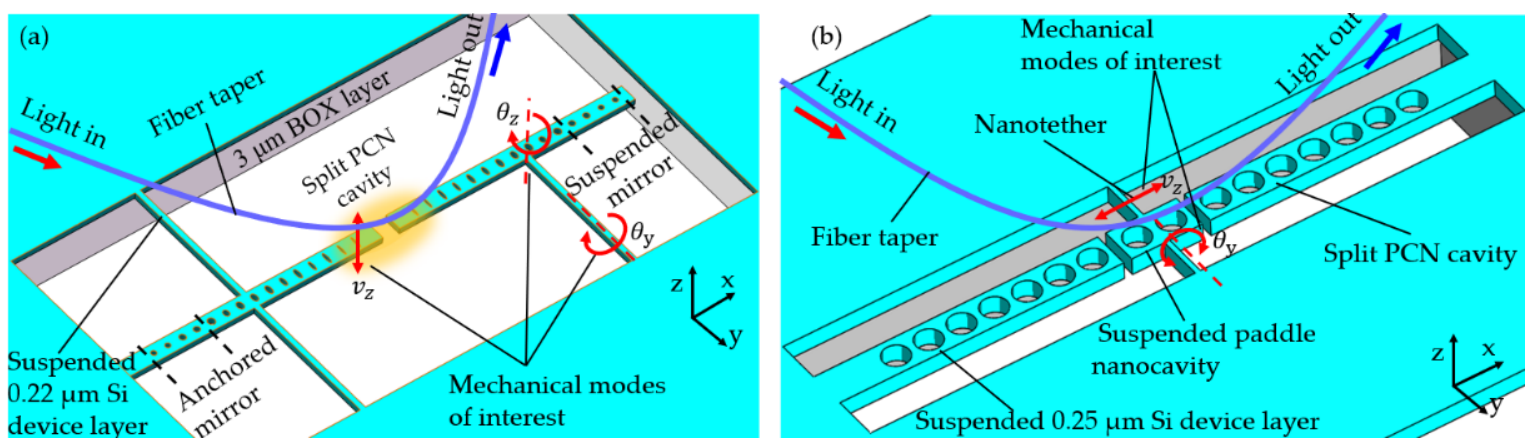

Figure 19. PCN nanocavity torque sensors: (a) photonic crystal split-beam nanocavity [124]; (b) photonic crystal paddle optomechanical cavity [126].

These optomechanical nanocavity systems provide a unique platform for studying optomechanical couplings and their impacts on magneto-mechanical sensing. When combining with magnetostrictive materials, this split PCN cavity can be used as an external magnetic field sensor. As presented in Figure 20, a magnetometer was proposed by integrating a ferromagnetic thin-film permalloy island onto a rectangular pad at the end of the moving nanobeam [70]. In the presence of an in-plane magnetic field $H_{x}$, the permalloy came to be magnetized with a net moment along the field $x$-direction. When a magnetic field $H_{z}$ directed along the $z$-direction, a magnetic torque was generated along the torsion rod that supported the moving nanobeam. Due to its torque sensitivity of $1.3 \times 10^{-21} \mathrm{Nm} / \sqrt{\mathrm{Hz}}$, the optomechanical nanocavity could detect nanoscale magnetic phenomena at the field strengths on the order of the earth's field (44-60 $\mu \mathrm{T})$, corresponds to a magnetic moment sensitivity of $(2.4 \pm 0.4) \times 10^{7} \mu_{\mathrm{B}}$.
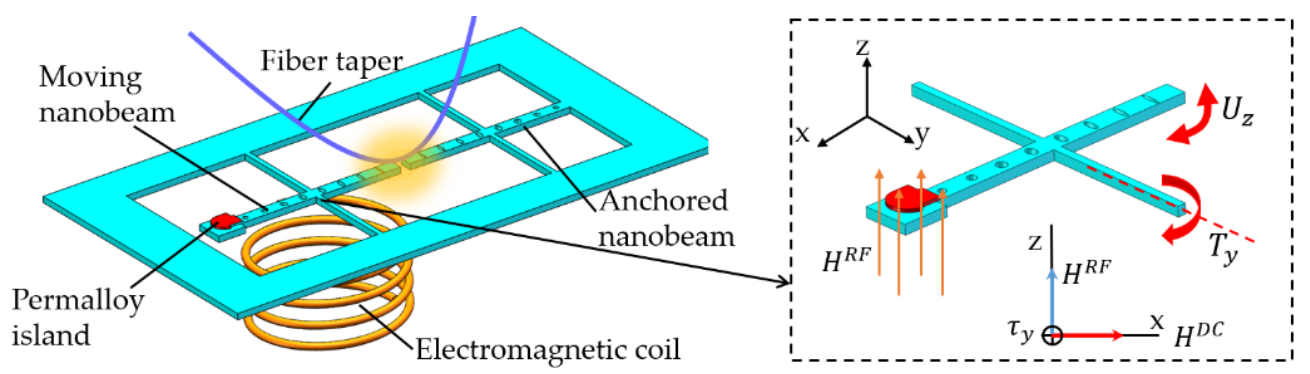

Figure 20. Split-beam PhC nanocavity for magnetic sensing (inset: mechanical profile of moving nanobeam under applied magnetic field $\mathrm{Hz}$ ) [70].

Another novel multi-cavity optomechanical torque sensor [127] using a nanobeam composed of two PCN cavities on each side was demonstrated as depicted in Figure 21a. The nanobeam was suspended on two axial spokes that were anchored to the substrate. In the architecture, the nanobeam could oscillate as a torsion driven by optical force excited in the side PCN cavity. Through optomechanical modulation of coupling between the two PCN cavities in the unresolved side-band regime, optical force generated by photons inside the PCN cavities leads to rotation of the nanobeam. The mechanical rotation of optomechanical nanobeam modulated two PCN cavities dispersively in an antisymmetric way, as shown in Figure $21 \mathrm{~b}$. The nanobeam rotated counter-clockwise under the blue-shifted pump pulse and rotated clockwise under the red-shifted pump pulse. Finally, a multi-cavity optomechanical torsion sensor was demonstrated with a very high detection sensitivity of the torque moment determined to be $9.7 \times 10^{-21} \mathrm{~N} \cdot \mathrm{m} / \mathrm{Hz}^{1 / 2}$ and could be potentially utilized in some reported torsional magnetometer [128,129] and accelerometer [130]. 

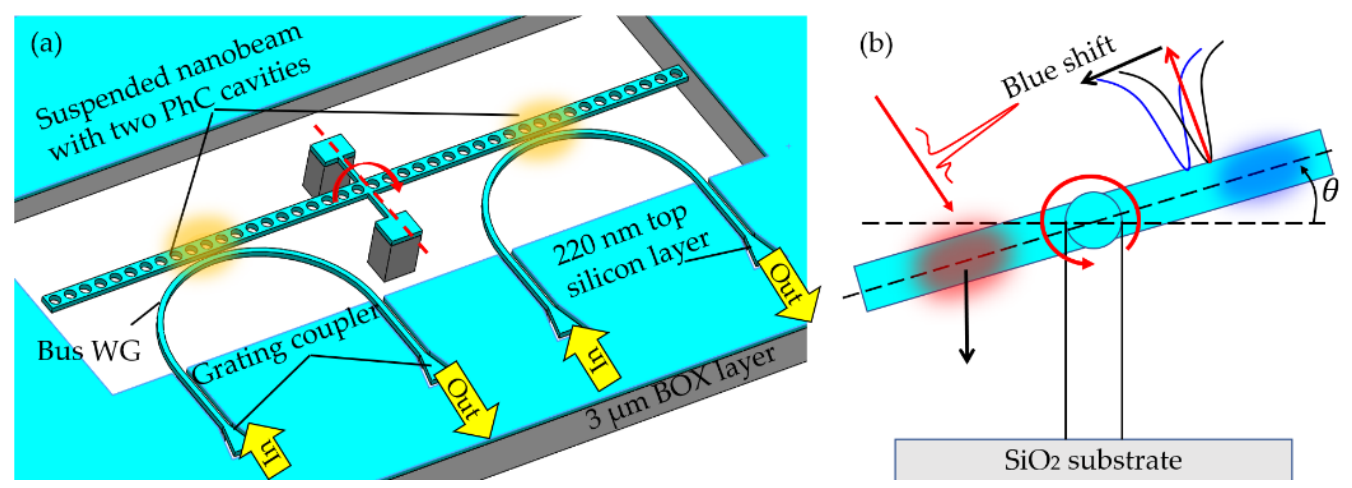

Figure 21. Optomechanical torsion sensor: (a) a suspended nanobeam inscribed with PhC cavities at each side; (b) laser detuning of mechanical twisting of nanobeam [127].

\subsubsection{Mass Sensing}

Mass sensors play a significant role in the nanotechnological measurements. Traditionally, the mass spectrometer is used for nanoparticle sensing, but the particle should be ionized before extraction. In recent years, nanomechanical cavities are widely applied in mass detection with a high-mass sensitivity of as small as $1 \times 10^{-21} \mathrm{~g}$ in vacuum and $1 \times 10^{-15} \mathrm{~g}$ in liquid condition. With the mass variation $\Delta m$ deposited onto the surface of a nanomechanical cavity, this can lead to the resonant frequency shift given by $\Delta m=\frac{2 m_{e f f}}{\Omega_{m}} \Omega_{m}$. To realize high mass sensitivity, it can be clearly seen that resonator mass $m_{\text {eff }}$ should be small but both mechanical frequency $\Omega_{m}$ and Q-factor $Q_{m}$ should be large to obtain a small minimum detectable frequency. Some efforts $[108,131,132]$ using cavity optomechanics for mass sensing have been reported. However, thermo-optical noise caused by the instability of coupling methods, for example tapered fiber coupling, limits their sensing resolution to the level of picograms. To reduce this noise, the integrated bus waveguide [133] coupling may be an alternative and effective scheme. For PhC cavities based optomechanical mass sensors, most research has focused on the numerical analysis [134-136], as shown in Figure 22a. Theoretically, any mass change in the sensing oscillator could cause a certain amount of mechanical frequency shift. When a nanoparticle deviated from the center of the crystal hole, the optical trapping force and optical potential on the manipulated nanoparticle were analyzed. As the radius of particle varied from 100 to $500 \mathrm{~nm}$, the mechanical frequency of particle decreased from 4.6 to $0.9 \mathrm{GHz}$. Significantly, the corresponding changing percent of the mechanical frequency of the cavity, $\Delta \Omega_{m} / \Omega_{m}$ reached up to approximately $400 \%$. Compared with the optical frequency shift of $\pm 0.06 \%$, monitoring the mechanical frequency was demonstrated as the better performance for particle sensing. However, the experimental demonstration of these sensing concepts was limited by the fiber taper coupling configuration and the quantification of nanoparticles. As a result, an alternative coupling method [137] in a PCN resonator was experimentally proposed as shown in Figure 22b. An on-chip femtogram-scale optomechanical crystal nanobeam with a coupled bus waveguide was operated in air and water environments. With a drop in mechanical quality factor from 2285 in the air to 6.6 in the water, the mechanical frequency was reduced to 5.251 from $5.3 \mathrm{GHz}$ in air. Finally, the mass sensitivity of this proposed device could be estimated to be at the level of $1.33 \mathrm{ag} / \sqrt{\mathrm{Hz}}$. To be noted, these femtogram-scale nano-optomechanical resonators in liquid were promising in chemical analysis and biomedical applications.

Meanwhile, nanomechanical resonators have extremely high sensitivities due to their ultrasmall mass and large mechanical Q-factor. For nanomechanical cavity mass sensing, the minimum detectable mass resolution is determined by the effective mass of a mechanical resonator. Therefore, another solution to further enhance mass sensitivity is to introduce a nanomechanical resonator into the on-chip integrated optomechanical systems $[83,86,138]$. As shown in Figure 23, a femtogram scale nanomechanical resonator with an ultralow effective mass of $6.42 \mathrm{fg}$ was embedded in a double-slot PCN cavity [138] with a measured optical Q-factor up to $2.25 \times 10^{4}$. A displacement sensitivity of $0.13 \mathrm{fm} / \mathrm{Hz}^{1 / 2}$ was demonstrated, which was 22 times beyond SQL. Similarly, a flexural nanomechanical 
resonator was also demonstrated in a 2D PhC nanocavity [83]. The doubly clamped nanomechanical resonators with mass as small as $25 \mathrm{fg}$ were embedded in the middle of a 2D PhC nanocavity. Due to the strong optomechanical interaction in the nanoscale slot nanobeam cavity, a displacement sensitivity of $0.94 \mathrm{fm} / \sqrt{\mathrm{Hz}}$ was realized. Therefore, this scheme was favorable for the high-resolvable mass sensor due to its reduced mass of the resonator itself.
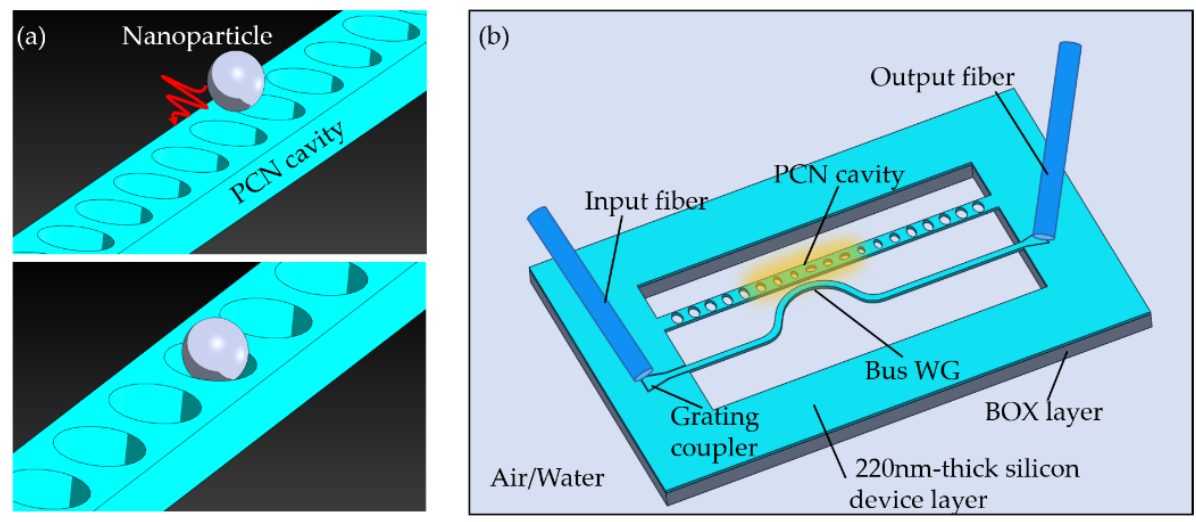

Figure 22. (a) Analytical model of PCN cavity for nanoparticle trapping [134-136]; (b) optomechanical $\mathrm{PhC}$ cavity operation in water for mass sensing (the colored box indicates that the optomechanical device is immersed in water) [137].

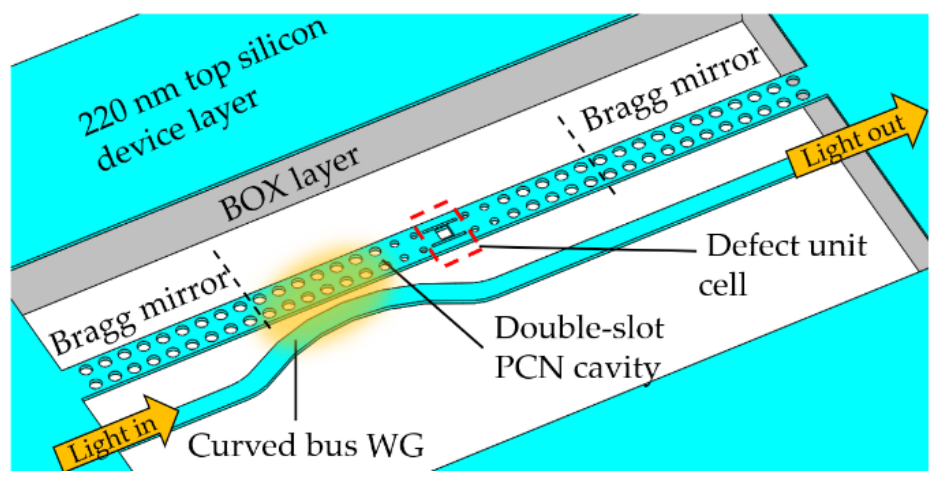

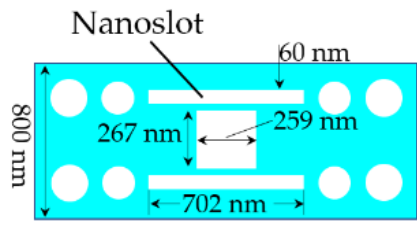

Central defect unit cell

Figure 23. A femtogram scale nanomechanical resonator embedded in a double-slot PCN cavity, and the right inset is zoom-in view of central nanomechanical resonator [138].

\subsubsection{Inertial Sensing}

Inertial sensors are generally used to detect and measure acceleration, tilt, and vibration. In principle, the MEMS-based accelerometer works to measure the displacement of a mechanical mass, known as a proof mass $[139,140]$. In MEMS devices, displacement detection is electrically measured through the capacitance between proof mass and electrode. However, currently available MEMS accelerometers still lack the sensitivity and bandwidth required for precise navigation, and the sensitivity is limited by thermal-electronic noise in the readout circuits. To eliminate the electronic noise effect, optical fiber sensing methods can reach the fundamental thermal-mechanical limit. In this critical limit, Brownian-motion of mechanical resonator limits the detection sensitivity. This thermal-mechanical noise imposes a trade-off between the sensitivity $\left(a_{t h}\right)$ and bandwidth $\left(\Omega_{m}\right)$ of the accelerometer given as $a_{t h}=\sqrt{4 \Omega_{m} k_{B} T / m Q_{m}}$. To achieve high sensitivity for a given bandwidth, the denominator term $m Q_{m}$ needs to be maximized. For instance, a sensitivity of $1 \mathrm{ng} / \mathrm{Hz}^{1 / 2}$ over a bandwidth of $10 \mathrm{kHz}$ requires $m Q_{m}>1 \mathrm{~kg}$. However, for high bandwidth devices, a high-resolution displacement measurement $x(w) \sim a_{t h} / \Omega_{m}^{2}$ at the fundamental mechanical frequency is required to approach the thermal limit. This technical task is difficult for electrical circuit readout, for example, MEMS accelerometers have $\mathrm{kHz}$ scale bandwidths, but their sensitivities are limited to a few $\mathrm{mg} / \mathrm{Hz}^{1 / 2}$. 
A comparison of sensitivity and bandwidth of three types of accelerator above has been summarized in Table 4.

Table 4. A comparison of sensitivity versus bandwidth of three representative accelerators.

\begin{tabular}{ccccc}
\hline Types of Accelerator & Device Size & Bandwidth & Sensitivity & Reference \\
\hline MEMS & Large $(\mathrm{mm}-\mathrm{scale})$ & $3.2-20 \mathrm{kHz}$ & $\sim 1 \mathrm{mg} / \mathrm{Hz}^{1 / 2}$ & {$[139,140]$} \\
Fiber optics & Small $(\mu \mathrm{m}-\mathrm{scale})$ & $1 \mathrm{kHz}$ & $0.1 \mu \mathrm{Hg} / \mathrm{Hz}^{1 / 2}$ & {$[141]$} \\
Optomechanics & Ultrasmall $(\mathrm{nm}-\mathrm{scale})$ & $>10 \mathrm{kHz}$ & $1-100 \mathrm{ng} / \mathrm{Hz}^{1 / 2}$ & {$[130,142]$} \\
\hline
\end{tabular}

As mentioned above, acceleration sensing is based on displacement sensing, and it prefers low mechanical frequency to achieve high sensitivity. Besides, its sensitivity is proportional to $g_{o m}$ because optomechanical coupling transduces mechanical motions to optical signals. As a result, many efforts in the optomechanical PhC cavities have been done to improve these two aspects. The first optomechanical accelerator was defined using an on-chip zipper PCN cavity to measure the motion of a large suspended $\mathrm{Si}_{3} \mathrm{~N}_{4}$ proof mass [130], as indicated in Figure 24. Two parallel suspended $\mathrm{Si}_{3} \mathrm{~N}_{4}$ doubly clamped PCNs (10- $\mu \mathrm{m}$-long and less than 1- $\mu \mathrm{m}$-wide), and a separation gap of around $100 \mathrm{~nm}$ formed the zipper cavity. Due to the high confinement of optical mode between the coupled nanobeams, the optical resonance frequency was extremely sensitive to the mechanical motion of moveable nanobeam cavity. To work as an accelerometer, a large proof mass was attached to the movable beams to realize the motion sensing. The detection could reach up to the standard quantum limit experimentally. Using the highly tensioned tethers supporting the proof-mass, a large proof mass can be realized without sacrificing mechanical $Q_{m}$, and hence reduce the effect of thermal noise. The proposed optomechanical accelerator exhibited a sensitivity of $\sim 0.1 \mu \mathrm{g} / \mathrm{Hz}^{1 / 2}$ near the mechanical resonance frequency around $20 \mathrm{kHz}$.

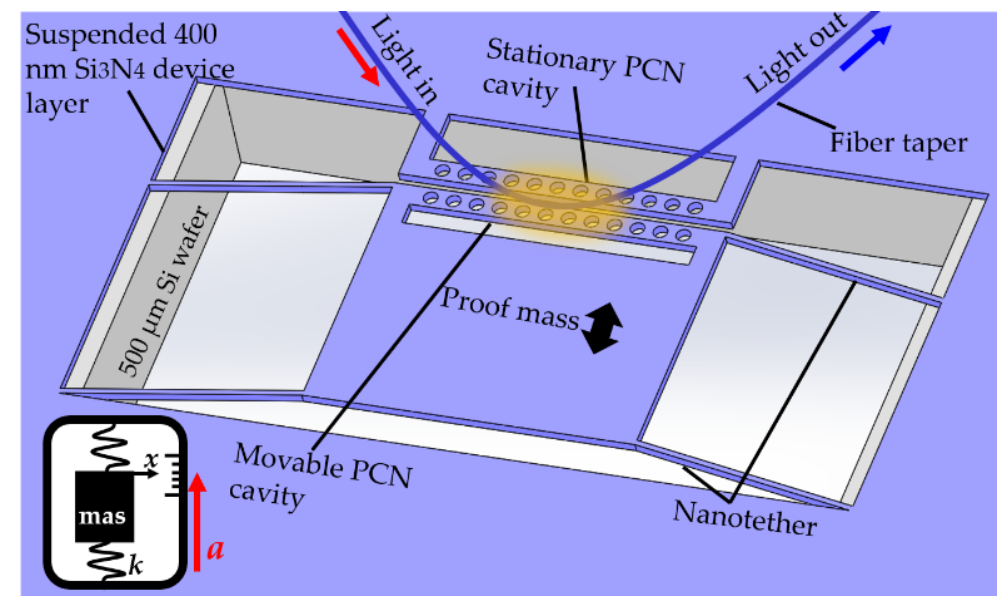

Figure 24. Schematic of PCN zipper cavity-based optomechanical accelerometer (the inset box indicates that canonical of accelerometer) [130].

In a similar manner, another optomechanical inertial sensor based on a slot-type 2D photonic crystal cavity [143] was demonstrated in Figure 25a. One side of the slot cavity was anchored on the silicon top layer and the other suspended side was attached to a large proof mass of $5.6 \mathrm{ng}$ for acceleration detection. When an acceleration was applied to the motional proof mass, the slot separation width was perturbed to shift the optical resonance frequency and change the optomechanical stiffness detuning from the driven laser frequency. It was demonstrated that this optomechanical inertial sensor provided an $8.2 \mu \mathrm{g} \mathrm{Hz}^{-1 / 2}$ velocity random walk at an acquisition rate of $100 \mathrm{~Hz}$ and an enhanced optical driving transduction sensitivity of $625 \mu \mathrm{g} \mathrm{Hz}$. What is more, an apparatus for detecting gravitational force $[144,145]$ was provided based on a similar identical scheme in Figure 25b. The 
optomechanical oscillator deformed under gravitational force, which caused a shift in resonance associated with the optomechanical sensitivity up to $10 \mathrm{ng} / \mathrm{Hz}^{-1 / 2}$ [144]. However, considering practical engineering applications, one large obstacle that should not be underestimated is associated with a complete integration on-chip device. Significant studies and engineering works are needed to achieve a compact integration system that not only contains the optomechanical device in a vacuum, but also the highly efficient light coupling, readout receiver and related frequency modulation.
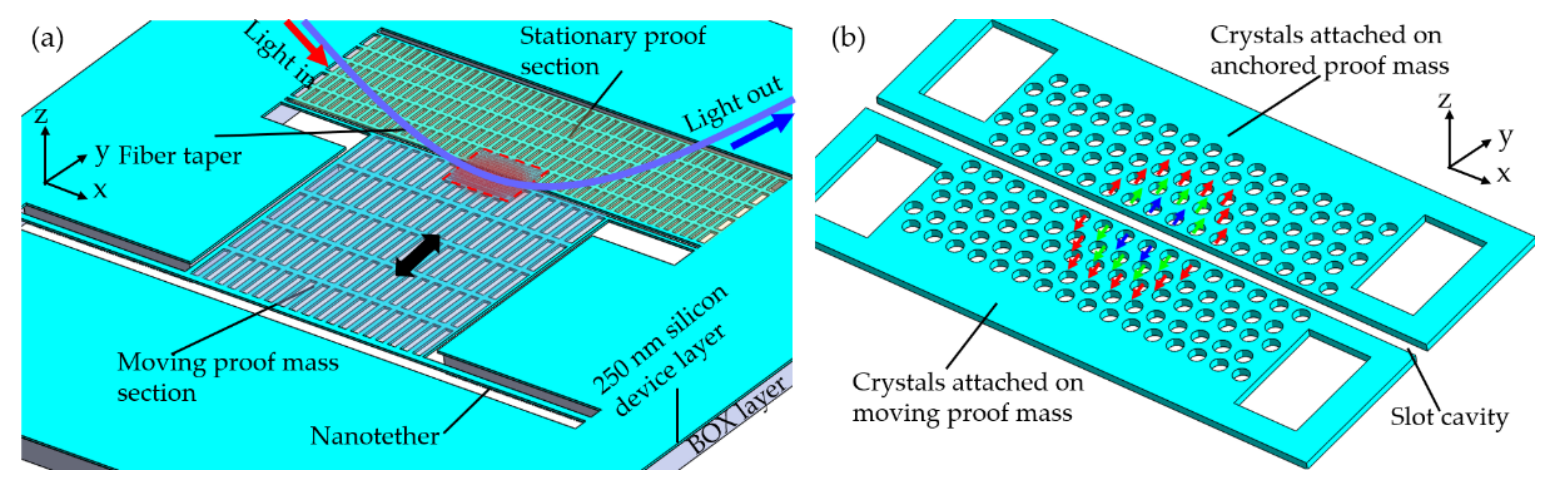

Figure 25. (a) Schematic of a chip-scale 2D PhC slot cavity for acceleration sensing; (b) close-up view of optomechanical cavity with colored arrows denoting three different photonic crystal lattice perturbations resulting in optomechanical stiffening [144,145].

\subsubsection{Optomechanical Detection on Angular Momentum of Light}

Photons carry linear momentum and spin angular momentum when they are circularly or elliptically polarized. In the light-matter interaction, the transfer of linear momentum creates optical forces, whereas transfer of angular momentum induces optical torque. A wealth of applications of photon angular momentum have been widely proposed. Methods for measuring photon angular momentum have been rapidly developed but rarely explored in integrated photonics. Recently, the photon angular momentum measurements were set out by using the on-chip optomechanical systems. For PhC optomechanical cavities, the first integrated optomechanical resonator to detect the spin angular momentum and optical torque [146] was proposed using a silicon nano-optomechanical device as shown in Figure 26. There was a suspended nanobeam in which two PCN nanocavities were embedded on each side and attached to a birefringent waveguide. The core element of the device was a birefringent suspended structure due to the angular momentum exchange between the light and the waveguide. As a result, the photons applied an optical torque on the birefringent waveguide to twist or float. The resonance modes of the nanocavities were particularly sensitive to their distance from the substrate, so they could provide very sensitive detection to the rotation of the waveguide actuated by the optical torque.

In addition, light with a helical wavefront also possess orbital angular momentum (OAM). As presented in Figure 27, an optomechanical PhC cavity to detect the optical torque allowed measurement of the OAM of light [147], as well as non-absorbing optical field detection via light's OAM degree of freedom. One of the nanobeams was attached to the central pad, while the other was fixed to the surrounding chip. Optical actuation of the central pad induced by OAM could shift the nanobeam's center of mass position through twisting of the central pad, and through excitation of the mechanical bouncing mode of nanobeam. The device had a torque sensitivity of $\tau_{\min }=3.22 \times 10^{-21} \mathrm{~N} \cdot \mathrm{m} / \mathrm{Hz}^{1 / 2}$, allowing OAM detection of optical fields with a sensitivity of $P_{\min }=8.7 \mu \mathrm{W} / \mathrm{Hz}^{1 / 2}$. Research on optical torque and optomechanical interplay with photon angular momentum can contribute to torsion-based cavity optomechanics and photon spin-orbit optomechanical coupling, as well as sensing applications such as gyroscope and torsional magnetometry. 


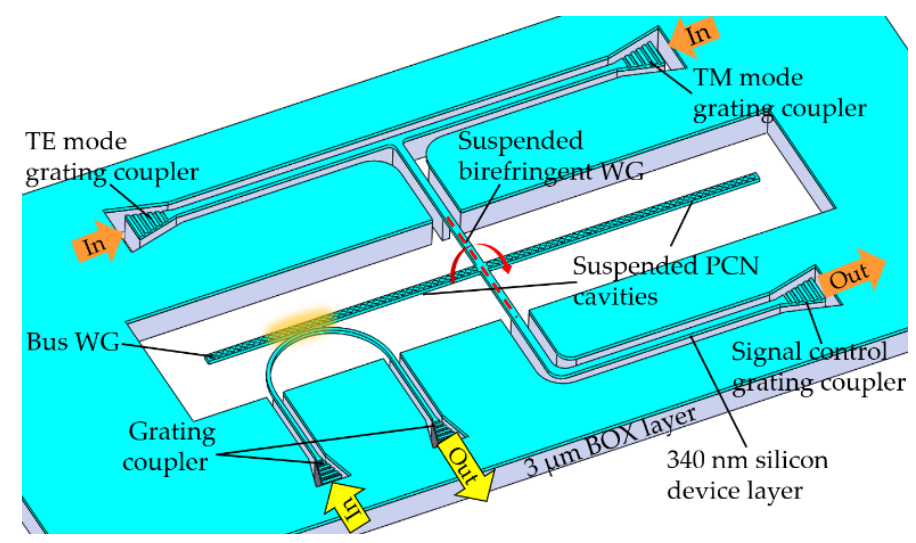

Figure 26. Optomechanical spin angular momentum sensor using a suspended waveguide-nanobeam structure [146].

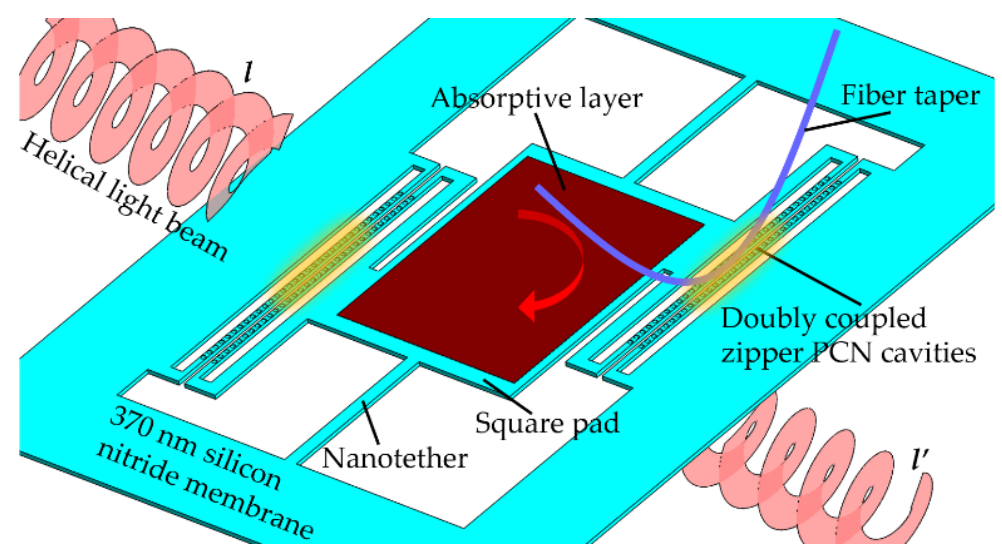

Figure 27. Optomechanical orbital angular momentum (OAM) sensor using a pair of zipper cavities [148].

\subsubsection{Summary of Radiation Pressure-Driven PhC Cavity Sensors}

In the previous discussion, we have reviewed most of the typical PhC cavities for radiation pressure-driven sensing applications based on the types of target signals to measure. In Table 5, some significant parameters of representative works are summarized in the views of experimental condition and optomechanical characteristics. Several conclusions can be derived from this table: first, silicon-based materials are frequently used in the integrated optomechanical devices due to their mature fabrication technology and feasible optical/mechanical properties in cavity design. Compared with silicon, silicon nitride expands the applications reaching up to a wavelength of $3.5 \mu \mathrm{m}$ with a larger electronic bandgap. Second, a vacuum condition is required in most studies on optomechanics. To eliminate or minimize the impact of air-damping on the mechanical resonator, a low-pressure vacuum condition can greatly enhance the mechanical quality factor. Therefore, this vacuum condition requirement raises a relatively rigorous condition for experimental optomechanical sensing applications. Another challenging work in the optomechanical device is the light coupling and collection architecture. It can be seen that tapered fiber is widely used in most studies due to its high coupling efficiency. However, in the practical fabrication process of dimpled tapered fiber, the operations of packaging and dimpling on the tapered fiber are so challenging that they could limit the development of integration on optomechanical photonics. In addition, due to its extremely flexible structure, the tapered fiber is usually placed and partially contacted with photonic resonator. In this case, the coupling between tapered fiber and optical cavity is hard to adjust for a high-performance optomechanical coupling. From the perspective of sensing applications, the above two points are obvious drawbacks in radiation pressure-driven $\mathrm{PhC}$ cavities. 
Table 5. Summary of selected experiments on radiation pressure-driven $\mathrm{PhC}$ sensors.

\begin{tabular}{|c|c|c|c|c|c|c|c|c|}
\hline \multirow[b]{2}{*}{ Cavity Type } & \multirow[b]{2}{*}{$\begin{array}{c}\text { Material } \\
(\mathrm{Opt}+\mathrm{Mech})^{\mathrm{a}}\end{array}$} & \multicolumn{2}{|c|}{ Experiment Condition } & \multirow[b]{2}{*}{$m_{e f f} / g$} & \multirow[b]{2}{*}{$\Omega_{m} / \mathrm{Hz}$} & \multirow[b]{2}{*}{$Q_{m}$} & \multirow[b]{2}{*}{$Q_{o p t}$} & \multirow[b]{2}{*}{$\begin{array}{c}\text { Sensing } \\
\text { Application }\end{array}$} \\
\hline & & $\begin{array}{l}\text { Vacuum } \\
(\mathrm{Y} / \mathrm{N})\end{array}$ & $\begin{array}{l}\text { Coupling } \\
\text { Scheme }\end{array}$ & & & & & \\
\hline PCN & $\mathrm{Si}+\mathrm{Si}{ }^{\mathrm{b}}$ & $\mathrm{N}$ & Tapered fiber & $1.2 \times 10^{-12 *}$ & $4.4 \times 10^{9}$ & 2900 & $3.8 \times 10^{4}$ & RI [109] \\
\hline Coupled PCN & $\mathrm{SiN}+\mathrm{SiN}^{\mathrm{c}}$ & $\mathrm{Y}$ & Tapered fiber & $1.0 \times 10^{-8}$ & $2.7 \times 10^{4}$ & $1.4 \times 10^{4}$ & 9500 & Acceleration [130] \\
\hline PhC zipper & $\mathrm{SiN}+\mathrm{SiN}$ & $\mathrm{N}$ & Tapered fiber & $4.3 \times 10^{-11}$ & $8 \times 10^{6}$ & $50 \sim 150$ & $3 \times 10^{5}$ & Motion [45] \\
\hline Split PCN & $\mathrm{Si}+\mathrm{Si}$ & $\mathrm{N}$ & Tapered fiber & $1.0 \times 10^{-12}$ & $3 \times 10^{6}$ & $<100$ & 5000 & Magnetometry [70] \\
\hline $\begin{array}{l}\text { Beam embedded } \\
\text { 2D PhC }\end{array}$ & $\mathrm{Si}+\mathrm{Si}$ & $\mathrm{Y}$ & $\begin{array}{l}\text { Grating } \\
\text { coupler }\end{array}$ & $2.5 \times 10^{-12}$ & $6.3 \times 10^{9}$ & 1230 & $2 \times 10^{4}$ & Mass [86] \\
\hline Air-slot 2D PhC & $\mathrm{Si}+\mathrm{Si}$ & $\mathrm{Y}$ & Tapered fiber & $2 \times 10^{-11}$ & $1.5 \times 10^{8}$ & $1500 *$ & $1.2 \times 10^{6}$ & Displacement [85] \\
\hline
\end{tabular}

\subsection{Future Perspectives for Optomechanical Sensing Based on PhC Cavities}

\subsubsection{Nonlinear Optomechanics Enhanced Sensitivity}

In most optomechanical interaction, the solution for optomechanical systems is obtained using an approximately linearized method. However, nonlinear effects are conceptually important and cause bistable behavior in the classical regime, which has a non-negligible effect on the optomechanical system $[17,34]$. There are two sources of nonlinear behavior in cavity optomechanics. First, the radiation pressure interaction is inherently nonlinear under high-intensity light input. Plenty of theoretical proposals [148-150] and experimental demonstrations [121,151-154] use the radiation pressure-driven motion of mechanical resonator to generate the second-order sideband in the optomechanical system. In this case, the mechanical resonator is coupled to the optical cavity at resonant frequency through shifts in the effective index of refraction as $\omega_{c a v} \rightarrow \omega_{c a v}-g_{o m} x-g_{2} x^{2}$. Therefore, the interaction Hamiltonian including the second-order optomechanical coupling can be rewritten as [121],

$$
\hat{H}_{\text {int }}=-\hbar\left(g_{o m} x+g_{2} x_{2}\right) \hat{a} \hat{a}
$$

where, $g_{2}=-1 / 2 \partial^{2} \omega_{c a v} / \partial x^{2}$. In the mechanical spectrum, the peak of mechanical displacement term $x^{2}$ arising from the second-order optomechanical interaction occurs at the double and harmonized fundamental mechanical resonant frequency. Except for the nonlinear effect induced by the second-order optomechanical coupling, another direct approach is to induce an inherently nonlinear mechanical resonator. Generally, nonlinear mechanical resonators are presented with low-dimensional resonators such as carbon nanotubes and graphene flakes $[155,156]$. Here, nonlinear effects in the optomechanical system are investigated by coupling a radiation pressure to a nonlinear mechanical resonator. Referring to Equation (5) in the linearized optomechanical system, the quantum Langevin equations for optical and mechanical modes in the nonlinear system should be derived as [157],

$$
\left\{\begin{array}{c}
\dot{\alpha}=-\frac{\kappa}{2} \alpha+i(\Delta+G x) \alpha+\sqrt{\kappa_{e x}} \alpha_{i n} \\
m_{e f f} \ddot{x}=-m_{e f f} \Omega_{m}^{2} x-m_{e f f} \Gamma_{m} \dot{x}+\hbar G|\alpha|^{2}+\alpha x^{3}
\end{array}\right.
$$

where, the mechanical nonlinearity is expressed by the Duffing term with a strength $\alpha$. These nonlinear mechanical effects can be investigated using an important tool, namely, optomechanically induced transparency (OMIT) $[158,159]$ to determine the optomechanical coupling qualitatively. The shape of the OMIT dip behaves similarly with the frequency response in the nonlinear (Duffing) oscillator and exhibits bistability with a hysteretic behavior. Considering the nonlinear optomechanical effects for sensing applications, the presence or variation of an environmental parameter, such as refractive index or movable dielectric boundary, can be converted to the optical resonance frequency and eventually affects the transmission power in the steady-state. An overall sensitivity $(S)$ is defined by the ratio of the varying optical transmission to the environmental perturbation [160] as, 


$$
S=\frac{d T}{d \Delta} \cdot \frac{d \Delta}{d n} \cdot \frac{d n}{d \alpha}
$$

where $T=P_{\text {out }} / P_{\text {in }}$ is the optical transmission and $d \alpha$ indicates the change of environmental parameter. In most resonators, the derivative of frequency detuning over mode index $(d \Delta / d n)$ depend on the refractive index and geometry of the cavity and this variation is generally small. Similarly, the transduction of external perturbation to varying mode index $(d n / d \alpha)$ is also small. Since the last two parts are fixed for a given resonator, alternatively, further increase in the first term $d T / d \Delta$ can enhance the total sensitivity. Considering that nonlinear optomechanics induced resonance shift, the transmission curve becomes asymmetric. Based on the concept of nonlinear optomechanical sensor, the optomechanical effect is used to engineer the transmission spectrum and increase its slope. Owing to the overall sensitivity is proportional to the slope, it has been demonstrated that dispersive optomechanical coupling can enhance sensitivity by at least two orders of magnitude [160].

For engineering applications based on nonlinear effects in the optomechanical systems, in addition to introducing the second order optomechanical coupling for enhanced sensitivity in precision sensing for displacement [117-119,152], several nonlinear optomechanical models have been developed and analyzed for magnetic field [161,162], and electrical charge [163-167] sensing recently. Another proposal concept is the integration of a nonlinear mechanical resonator into cavity optomechanics. For instance, in a doubly coupled zipper PCN cavity, the movable nanobeam can be anchored with a nonlinear mechanical part. Typically, a curved bistable beam bucking could be a possible candidate for this nonlinear mechanical resonator [168]. When the optomechanical coupling occurs, the nonlinear effects from mechanical oscillators give rise to intense optical response and interesting dynamic backaction phenomena.

\subsubsection{Two-Dimensional Materials Integrated with Optomechanical Systems}

Besides conventional mechanical resonators, two dimensional materials [169], are also extensively explored to construct novel optomechanical systems. For instance, a simple patterned graphene layer can be engineered to control light confinement compared with that of dielectric materials. Graphene, a single layer of hexagonally packed carbon atoms, endows extraordinary electronic, optical, thermal, and mechanical properties [170-172]. Optical conductivity of graphene can be effectively modified by electrically controlling its Fermi level, which enables the optical absorption of graphene-based reconfigurable photonic devices. Besides, mechanical resonators based on tensed graphene membranes with a low mass, $\rho \approx 0.75 \mathrm{mg} / \mathrm{m}^{2}$, have been widely studied for force and mass sensing with a significant improvement in sensitivity. Therefore, feasible electrical integrability, and strong optical interaction, ultra-small mass and high operating frequencies of graphene makes it an attractive functional material to be integrated with micro-/nano-photonic devices [173-175]. Here, the conceptual proposal focuses on integration of graphene and on-chip PCN cavity for optomechanical applications.

Based on the optomechanical principles and graphene properties, the optomechanical graphene integrated PCN resonator is proposed in Figure 28. In order to maximize the optomechanical coupling between the silicon nanobeam and graphene in the graphene drum areas, an air-mode nanobeam is designed to confine optical resonant modes within the central air holes. The optical absorption of graphene covered on the silicon waveguide causes absorption loss. Hence it leads to a dramatical reduction of Q-factors [176] and severely affects the optomechanical coupling strength. One feasible solution is to define the geometry of graphene pattern (length $L_{g}$ ) and location of graphene coverage on nanobeam. In the reported graphene optomechanical systems [177,178], graphene itself acts as mechanical oscillator and it will interplay with the optical resonator. In this way, they two generate optomechanical phenomena by laser detuning. Another electro-opto-mechanical graphene-nanobeam resonator $[179,180]$ can also be conceptually designed by integrating electronic circuits. Under an electrostatic force generated by the electronic circuits, the deflection of graphene gives rise to tension and strain to modulate optomechanics in the graphene integrated PCN resonator. 
In addition, the concept of Fermi level modulation [181] for tuning of optical absorption and graphene micro-heater [182] can also be explored and applied to potential sensing applications based on opto-mechanical interaction.

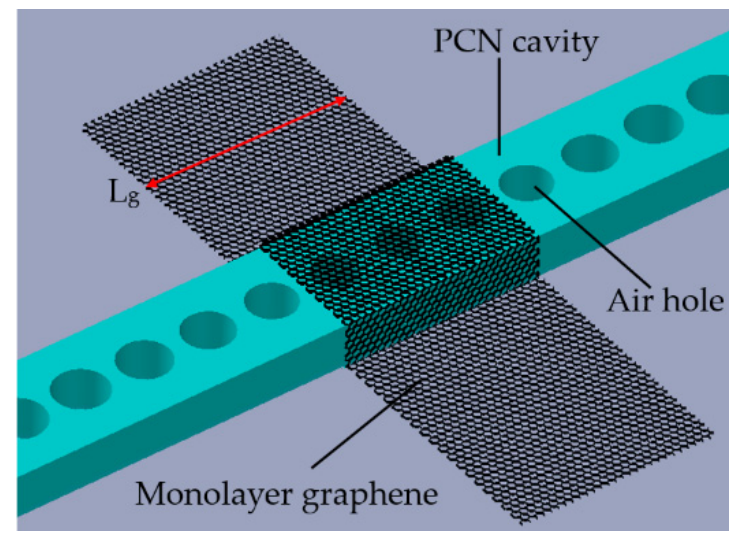

Figure 28. Proposal scheme of opto-mechanical PCN cavity with graphene integrated.

\section{Summary and Outlook}

As discussed above, on-chip tunable PhC devices integrated with N/MEMS generally have been demonstrated for various sensing applications on mechanical measurements. With further advancements in nanofabrication and device integration techniques, dynamically tunable $\mathrm{PhC}$ systems with ultra-precise features are expected to achieve a better sensing performance. A significant technical breakthrough in the mass production of a 5-nm semiconductor device comes out in 2020, which further enables the manufacturing ability of micro-nano on-chip sensors and networks sensors with smaller features and lower fabrication errors in future applications. Moreover, coupling techniques for the integrated on-chip device are the key and challenging factors to achieve practical opto-mechanical devices, playing an important role in sensing applications of PhCs.

For radiation pressure-driven $\mathrm{PhCs}$, as the field of cavity optomechanics is still under booming development, it is expanding rapidly in both basic physics and engineering applications. When it comes to sensing applications, cavity optomechanics possesses certain advantages and challenges. Compared with most N/MEMS devices with limited operational bandwidth, optomechanical PhC devices allow both optical actuation and readout to provide faster sampling and scanning rate. Besides, optomechanical devices can be operated at high frequencies up to $\mathrm{GHz}$, and thus they can directly link the signal domains at microwave frequencies in optical cavities for signal conversion $[60,183,184]$. In addition, optomechanical $\mathrm{PhC}$ cavities can offer the unique resource of strong optical backaction effects, which allows dynamic control of the mechanical resonance frequency and bandwidth. It should be noted that most realized optomechanical PhC systems till now have selected the device material with a large Young's modulus, such as silicon, silicon nitride and silica, etc., mainly considering their compatibility with nanofabrication technology and optical properties. However, the displacement of these materials driven by optical force is at most of the order of tens of $\mathrm{nm}$, thereby it always reduces their mechanical stiffness and affects optomechanical performances. To achieve larger sensitivity and tunability, it is meaningful to exploit mechanically compliant materials such as polymers $[185,186]$, which can reduce cost and can be easily combined with microfluidic platforms.

In summary, PhC cavities with the interplay of optical and mechanical resonators for sensing application are reviewed. The basic physics, experimental systems, and sensing applications in the context of mechanically tunable and radiation pressure-driven cavities are presented. Mechanically tunable PhC sensors have the characteristics of the simple system, easy fabrication, high precision, and dynamic control capability. Meanwhile, radiation pressure-driven PhC cavities reveal significant improvements in the performance of enhanced sensitivity and faster operation frequency. However, it also raises the stringent requirements for experimental testing, for example, vacuum environment and 
low adiabatic temperature are required in most optomechanical measurements. In the future, cavity optomechanics will bring a new interaction between the optics-mechanics interface. It embarks on the long journey towards miniaturization and integration of signal processing and on-chip devices for multi-function applications.

Author Contributions: J.X. conducted the review work and write this manuscript; Q.Q. and G.Z. (Guangcan Zhou) checked and edited language and formal; G.Z. (Guangya Zhou) and F.S.C. supervised this work. All authors have read and agreed to the published version of the manuscript.

Funding: This research was funded by MOE Tier2 project, grant number R-265-000-694-112.

Conflicts of Interest: The authors declare no conflict of interest.

\section{References}

1. Ma, J.; Povinelli, M.L. Applications of optomechanical effects for on-chip manipulation of light signals. Curr. Opin. Solid State Mater. Sci. 2012, 16, 82-90. [CrossRef]

2. Notomi, M. Manipulating light with strongly modulated photonic crystals. Rep. Prog. Phys. 2010, 73, 096501. [CrossRef]

3. Midolo, L.; Schliesser, A.; Fiore, A. Nano-opto-electro-mechanical systems. Nat. Nanotechnol. 2018, 13, 11-18. [CrossRef] [PubMed]

4. Lončar, M. Opto-mechanical interaction in flexible photonic crystals. In Proceedings of the Laser Science, Orlando, FL, USA, 6-10 October; p. LTh3G.1.

5. Du, H.; Chau, F.S.; Zhou, G. Mechanically-Tunable Photonic Devices with On-Chip Integrated MEMS/NEMS Actuators. Micromachines 2016, 7, 69. [CrossRef] [PubMed]

6. Hadzialic, S.; Kim, S.; Sarioglu, A.F.; Sudbø, A.S.; Solgaard, O. Displacement sensing with a mechanically tunable photonic crystal. IEEE Photonics Technol. Lett. 2010, 22, 1196-1198. [CrossRef]

7. Edinger, P.; Errando-Herranz, C.; Takabayashi, A.Y.; Sattari, H.; Quack, N.; Verheyen, P.; Bogaerts, W.; Gylfason, K.B. Compact low loss MEMS phase shifters for scalable field-programmable silicon photonics. In Proceedings of the CLEO: Science and Innovations, Washington, DC, USA, 10-15 May 2020; p. SM3J.2.

8. Chew, X.; Zhou, G.; Yu, H.; Chau, F.S.; Deng, J.; Loke, Y.C.; Tang, X. An in-plane nano-mechanics approach to achieve reversible resonance control of photonic crystal nanocavities. Opt. Express 2010, 18, 22232-22244. [CrossRef]

9. Yang, D.; Tian, H.; Ji, Y. Microdisplacement sensor based on high-Q nanocavity in slot photonic crystal. Opt. Eng. 2011, 50, 054402. [CrossRef]

10. Galeotti, F.; Seršić Vollenbroek, I.; Petruzzella, M.; Pagliano, F.; van Otten, F.W.; Zobenica, Ž.; Mohtashami, A.; Marnani, H.S.; van der Heijden, R.W.; Fiore, A. On-chip waveguide-coupled opto-electro-mechanical system for nanoscale displacement sensing. APL Photonics 2020, 5, 026103. [CrossRef]

11. Li, B.; Lee, C. NEMS diaphragm sensors integrated with triple-nano-ring resonator. Sens. Actuators A Phys. 2011, 172, 61-68. [CrossRef]

12. Lu, T.W.; Lee, P.T. Ultra-high sensitivity optical stress sensor based on double-layered photonic crystal microcavity. Opt. Express 2009, 17, 1518-1526. [CrossRef]

13. Rogers, D.J.; Papadakis, S.J.; Churchill, L.R.; Wong, C.W. Chip-Scale Optomechanical Magnetometer. U.S. Patent No. 9,897,666, 20 February 2018.

14. Du, H.; Zhou, G.; Zhao, Y.; Chen, G.; Chau, F.S. Magnetic field sensor based on coupled photonic crystal nanobeam cavities. Appl. Phys. Lett. 2017, 110, 061110. [CrossRef]

15. Sun, S.; Kim, H.; Solomon, G.S.; Waks, E. Strain tuning of a quantum dot strongly coupled to a photonic crystal cavity. Appl. Phys. Lett. 2013, 103, 151102. [CrossRef]

16. Rogers, B.; Gullo, N.L.; De Chiara, G.; Palma, G.M.; Paternostro, M. Hybrid optomechanics for quantum technologies. Quantum Meas. Quantum Metrol. 2014, 2, 11-43. [CrossRef]

17. Aspelmeyer, M.; Kippenberg, T.J.; Marquardt, F. Cavity optomechanics. Rev. Mod. Phys. 2014, 86, 1391. [CrossRef]

18. Liu, Y.-C.; Xiao, Y.-F.; Luan, X.; Gong, Q.; Wong, C.W. Coupled cavities for motional ground-state cooling and strong optomechanical coupling. Phys. Rev. A 2015, 91, 033818. [CrossRef] 
19. Teufel, J.D.; Donner, T.; Li, D.; Harlow, J.W.; Allman, M.S.; Cicak, K.; Sirois, A.J.; Whittaker, J.D.; Lehnert, K.W.; Simmonds, R.W. Sideband cooling of micromechanical motion to the quantum ground state. Nature 2011, 475, 359-363. [CrossRef]

20. Teufel, J.D.; Donner, T.; Castellanos-Beltran, M.; Harlow, J.W.; Lehnert, K.W. Nanomechanical motion measured with an imprecision below that at the standard quantum limit. Nat. Nanotechnol. 2009, 4, 820-823. [CrossRef]

21. Hu, Y.-W.; Xiao, Y.-F.; Liu, Y.-C.; Gong, Q. Optomechanical sensing with on-chip microcavities. Front. Phys. 2013, 8, 475-490. [CrossRef]

22. Metcalfe, M. Applications of cavity optomechanics. Appl. Phys. Rev. 2014, 1, 031105. [CrossRef]

23. Corbitt, T.; Ottaway, D.; Innerhofer, E.; Pelc, J.; Mavalvala, N. Measurement of radiation-pressure-induced optomechanical dynamics in a suspended Fabry-Perot cavity. Phys. Rev. A 2006, 74, 021802. [CrossRef]

24. Schliesser, A.; Kippenberg, T.J. Cavity optomechanics with whispering-gallery mode optical micro-resonators. In Advances in Atomic, Molecular, and Optical Physics; Elsevier: Amsterdam, The Netherlands, 2010; Volume 58, pp. 207-323.

25. Kim, S.H.; Lee, K.-D.; Kim, J.-Y.; Kwon, M.-K.; Park, S.-J. Fabrication of photonic crystal structures on light emitting diodes by nanoimprint lithography. Nanotechnology 2007, 18, 055306. [CrossRef]

26. Thorhauge, M.; Frandsen, L.H.; Borel, P.I. Efficient photonic crystal directional couplers. Opt. Lett. 2003, 28, 1525-1527. [CrossRef] [PubMed]

27. Threm, D.; Nazirizadeh, Y.; Gerken, M. Photonic crystal biosensors towards on-chip integration. J. Biophotonics 2012, 5, 601-616. [CrossRef]

28. Goyal, A.K.; Dutta, H.S.; Pal, S. Recent advances and progress in photonic crystal-based gas sensors. J. Phys. D: Appl. Phys. 2017, 50, 203001. [CrossRef]

29. Qiao, Q.; Xia, J.; Lee, C.; Zhou, G. Applications of Photonic Crystal Nanobeam Cavities for Sensing. Micromachines 2018, 9, 541. [CrossRef] [PubMed]

30. Yang, D.Q.; Duan, B.; Liu, X.; Wang, A.Q.; Li, X.G.; Ji, Y.F. Photonic Crystal Nanobeam Cavities for Nanoscale Optical Sensing: A Review. Micromachines 2020, 11, 72. [CrossRef] [PubMed]

31. Kippenberg, T.J.; Vahala, K.J. Cavity optomechanics: Back-action at the mesoscale. Science 2008, 321, 1172-1176. [CrossRef]

32. Yong-Chun, L.; Yu-Wen, H.; Wei, W.C.; Yun-Feng, X. Review of cavity optomechanical cooling. Chin. Phys. B 2013, 22, 114213.

33. Favero, I.; Marquardt, F. Focus on optomechanics. New J. Phys. 2014, 16, 085006. [CrossRef]

34. Xiong, H.; Si, L.; Lv, X.; Yang, X.; Wu, Y. Review of cavity optomechanics in the weak-coupling regime: From linearization to intrinsic nonlinear interactions. Sci. China Phys. Mech. Astron. 2015, 58,1-13. [CrossRef]

35. Hossein-Zadeh, M.; Vahala, K.J. An optomechanical oscillator on a silicon chip. IEEE J. Sel. Top. Quantum Electron. 2009, 16, 276-287. [CrossRef]

36. Liu, Y.-1.; Wang, C.; Zhang, J.; Liu, Y.-x. Cavity optomechanics: Manipulating photons and phonons towards the single-photon strong coupling. Chin. Phys. B 2018, 27, 024204. [CrossRef]

37. Zhao, Y.; Zhang, Y.-N.; Wang, Q.; Hu, H. Review on the optimization methods of slow light in photonic crystal waveguide. IEEE Trans. Nanotechnol. 2015, 14, 407-426. [CrossRef]

38. Dutta, H.S.; Goyal, A.K.; Srivastava, V.; Pal, S. Coupling light in photonic crystal waveguides: A review. Photonics Nanostruct.-Fundam. Appl. 2016, 20, 41-58. [CrossRef]

39. Notomi, M.; Kuramochi, E.; Taniyama, H. Ultrahigh-Q nanocavity with 1D photonic gap. Opt. Express 2008, 16, 11095-11102. [CrossRef]

40. Song, B.-S.; Noda, S.; Asano, T.; Akahane, Y. Ultra-high-Q photonic double-heterostructure nanocavity. Nat. Mater. 2005, 4, 207-210. [CrossRef]

41. Ogawa, S.; Imada, M.; Yoshimoto, S.; Okano, M.; Noda, S. Control of light emission by 3D photonic crystals. Science 2004, 305, 227-229. [CrossRef]

42. Chew, X.; Zhou, G.; Chau, F.S.; Deng, J. Enhanced resonance tuning of photonic crystal nanocavities by integration of optimized near-field multitip nanoprobes. J. Nanophotonics 2011, 5, 059503. [CrossRef]

43. Chan, J.; Safavi-Naeini, A.H.; Hill, J.T.; Meenehan, S.; Painter, O. Optimized optomechanical crystal cavity with acoustic radiation shield. Appl. Phys. Lett. 2012, 101, 081115. [CrossRef] 
44. Tian, F.; Sumikura, H.; Kuramochi, E.; Taniyama, H.; Takiguchi, M.; Notomi, M. Optomechanical oscillator pumped and probed by optically two isolated photonic crystal cavity systems. Opt. Express 2016, 24, 28039-28055. [CrossRef]

45. Eichenfield, M.; Camacho, R.; Chan, J.; Vahala, K.J.; Painter, O. A picogram-and nanometre-scale photonic-crystal optomechanical cavity. Nature 2009, 459, 550-555. [CrossRef] [PubMed]

46. Hryciw, A.C.; Wu, M.; Khanaliloo, B.; Barclay, P.E. Tuning of nanocavity optomechanical coupling using a near-field fiber probe. Optica 2015, 2, 491-496. [CrossRef]

47. Leijssen, R.; Verhagen, E. Strong optomechanical interactions in a sliced photonic crystal nanobeam. Sci. Rep. 2015, 5, 15974. [CrossRef] [PubMed]

48. Schneider, K.; Seidler, P. Strong optomechanical coupling in a slotted photonic crystal nanobeam cavity with an ultrahigh quality factor-to-mode volume ratio. Opt. Express 2016, 24, 13850-13865. [CrossRef] [PubMed]

49. Chew, X.; Zhou, G.; Chau, F.S.; Deng, J.; Tang, X.; Loke, Y.C. Dynamic tuning of an optical resonator through MEMS-driven coupled photonic crystal nanocavities. Opt. Lett. 2010, 35, 2517-2519. [CrossRef] [PubMed]

50. Tian, F.; Zhou, G.; Du, Y.; Chau, F.S.; Deng, J.; Teo, S.L.; Akkipeddi, R. Nanoelectromechanicalsystems-controlled bistability of double-coupled photonic crystal cavities. Opt. Lett. 2013, 38, 3394-3397. [CrossRef]

51. Tian, F.; Zhou, G.; Siong Chau, F.; Deng, J.; Akkipeddi, R. Measurement of coupled cavities' optomechanical coupling coefficient using a nanoelectromechanical actuator. Appl. Phys. Lett. 2013, 102, 081101. [CrossRef]

52. Tian, F.; Zhou, G.; Du, Y.; Chau, F.S.; Deng, J.; Tang, X.; Akkipeddi, R. Energy-efficient utilization of bipolar optical forces in nano-optomechanical cavities. Opt. Express 2013, 21, 18398-18407. [CrossRef]

53. Du, H.; Zhang, X.; Chen, G.; Deng, J.; Chau, F.S.; Zhou, G. Precise control of coupling strength in photonic molecules over a wide range using nanoelectromechanical systems. Sci. Rep. 2016, 6, 24766. [CrossRef]

54. Du, H.; Zhang, X.; Deng, J.; Zhao, Y.; Chau, F.S.; Zhou, G. Lateral shearing optical gradient force in coupled nanobeam photonic crystal cavities. Appl. Phys. Lett. 2016, 108, 171102. [CrossRef]

55. Tian, F.; Zhou, G.; Chau, F.S.; Deng, J. Torsional optical spring effect in coupled nanobeam photonic crystal cavities. Opt. Lett. 2014, 39, 6289-6292. [CrossRef] [PubMed]

56. Tian, F.; Zhou, G.; Du, Y.; Chau, F.S.; Deng, J.; Akkipeddi, R. Out-of-plane nanomechanical tuning of double-coupled one-dimensional photonic crystal cavities. Opt. Lett. 2013, 38, 2005-2007. [CrossRef] [PubMed]

57. Midolo, L.; Yoon, S.N.; Pagliano, F.; Xia, T.; van Otten, F.W.; Lermer, M.; Hofling, S.; Fiore, A. Electromechanical tuning of vertically-coupled photonic crystal nanobeams. Opt. Express 2012, 20, 19255-19263. [CrossRef] [PubMed]

58. Cui, X.; Zhang, W.; Zhang, J.; Le Roux, X.; Alonso-Ramos, C.; Vivien, L.; He, J.-J.; Cassan, E. Tailoring mode splitting and degeneracy in silicon triply resonant nanobeam cavities. JOSA B 2019, 36, 1267-1272. [CrossRef]

59. Grutter, K.E.; Davanço, M.I.; Balram, K.C.; Srinivasan, K. Invited Article: Tuning and stabilization of optomechanical crystal cavities through NEMS integration. APL Photonics 2018, 3, 100801. [CrossRef]

60. Deotare, P.B.; Bulu, I.; Frank, I.W.; Quan, Q.; Zhang, Y.; Ilic, R.; Loncar, M. All optical reconfiguration of optomechanical filters. Nat. Commun. 2012, 3, 846. [CrossRef]

61. Cohen, J.D.; Meenehan, S.M.; Painter, O. Optical coupling to nanoscale optomechanical cavities for near quantum-limited motion transduction. Opt. Express 2013, 21, 11227-11236. [CrossRef]

62. Camacho, R.M.; Chan, J.; Eichenfield, M.; Painter, O. Characterization of radiation pressure and thermal effects in a nanoscale optomechanical cavity. Opt. Express 2009, 17, 15726-15735. [CrossRef]

63. Perahia, R.; Cohen, J.; Meenehan, S.; Alegre, T.M.; Painter, O. Electrostatically tunable optomechanical "zipper" cavity laser. Appl. Phys. Lett. 2010, 97, 191112. [CrossRef]

64. Westendorp, S. Cavity Optomechanics in Vertically-Coupled Photonic Crystal Nanobeams and Membranes. Ph.D. Thesis, Eindhoven University of Technology, Eindhoven, The Netherlands, 2014.

65. Lin, T.; Tian, F.; Shi, P.; Chau, F.S.; Zhou, G.; Tang, X.; Deng, J. Design of mechanically-tunable photonic crystal split-beam nanocavity. Opt. Lett. 2015, 40, 3504-3507. [CrossRef]

66. Shi, P.; Du, H.; Chau, F.S.; Zhou, G.; Deng, J. Tuning the quality factor of split nanobeam cavity by nanoelectromechanical systems. Opt. Express 2015, 23, 19338-19347. [CrossRef] [PubMed]

67. Lin, T.; Zhang, X.; Zou, Y.; Chau, F.S.; Deng, J.; Zhou, G. Out-of-plane nano-electro-mechanical tuning of the Fano resonance in photonic crystal split-beam nanocavity. Appl. Phys. Lett. 2015, 107, 153107. [CrossRef] 
68. Tian, F.; Zhou, G.; Chau, F.S.; Deng, J.; Du, Y.; Tang, X.; Akkipeddi, R.; Loke, Y.C. Tuning of split-ladder cavity by its intrinsic nano-deformation. Opt. Express 2012, 20, 27697-27707. [CrossRef] [PubMed]

69. Miri, M.; Sodagar, M.; Eftekhar, A.; Mehrany, K.; Rashidian, B.; Adibi, A. Wideband tunable photonic crystal cavity with electrostatic actuation. In Proceedings of the IEEE Photonics Conference, Burlingame, CA, USA, 23-27 September 2012; pp. 266-267.

70. Wu, M.; Wu, N.L.; Firdous, T.; Fani Sani, F.; Losby, J.E.; Freeman, M.R.; Barclay, P.E. Nanocavity optomechanical torque magnetometry and radiofrequency susceptometry. Nat. Nanotechnol. 2017, 12, 127-131. [CrossRef] [PubMed]

71. Seidler, P.; Lister, K.; Drechsler, U.; Hofrichter, J.; Stoferle, T. Slotted photonic crystal nanobeam cavity with an ultrahigh quality factor-to-mode volume ratio. Opt. Express 2013, 21, 32468-32483. [CrossRef]

72. Li, B.; Hsiao, F.-L.; Lee, C. Configuration analysis of sensing element for photonic crystal based NEMS cantilever using dual nano-ring resonator. Sens. Actuators A Phys. 2011, 169, 352-361. [CrossRef]

73. Mai, T.T.; Hsiao, F.-L.; Lee, C.; Xiang, W.; Chen, C.-C.; Choi, W. Optimization and comparison of photonic crystal resonators for silicon microcantilever sensors. Sens. Actuators A Phys. 2011, 165, 16-25. [CrossRef]

74. Abdulla, S.C.; Kauppinen, L.; Dijkstra, M.; de Boer, M.J.; Berenschot, E.; de Ridder, R.; Krijnen, G.J. Micro-cantilever integrated 2D photonic crystal slab waveguide for enhanced dispersion tuning. J. Micromech. Microeng. 2011, 21, 125010. [CrossRef]

75. Gao, J.; McMillan, J.F.; Wu, M.-C.; Zheng, J.; Assefa, S.; Wong, C.W. Demonstration of an air-slot mode-gap confined photonic crystal slab nanocavity with ultrasmall mode volumes. Appl. Phys. Lett. 2010, 96, 051123. [CrossRef]

76. Pitanti, A.; Fink, J.M.; Safavi-Naeini, A.H.; Hill, J.T.; Lei, C.U.; Tredicucci, A.; Painter, O. Strong opto-electro-mechanical coupling in a silicon photonic crystal cavity. Opt. Express 2015, 23, 3196-3208. [CrossRef]

77. Mao, D.; Liu, P.; Ho, K.-M.; Dong, L. A theoretical study of a nano-opto-mechanical sensor using a photonic crystal-cantilever cavity. J. Opt. 2012, 14, 075002. [CrossRef]

78. Wang, Q.; Mao, D.; Dong, L. MEMS Tunable Photonic Crystal-Cantilever Cavity. J. Microelectromech. Syst. 2019, 28, 741-743. [CrossRef]

79. Midolo, L.; Pagliano, F.; Hoang, T.; Xia, T.; van Otten, F.; Li, L.; Linfield, E.; Lermer, M.; Höfling, S.; Fiore, A. Spontaneous emission control of single quantum dots by electromechanical tuning of a photonic crystal cavity. Appl. Phys. Lett. 2012, 101, 091106. [CrossRef]

80. Roh, Y.-G.; Tanabe, T.; Shinya, A.; Taniyama, H.; Kuramochi, E.; Matsuo, S.; Sato, T.; Notomi, M. Strong optomechanical interaction in a bilayer photonic crystal. Phys. Rev. B 2010, 81, 121101. [CrossRef]

81. Gavartin, E.; Braive, R.; Sagnes, I.; Arcizet, O.; Beveratos, A.; Kippenberg, T.J.; Robert-Philip, I. Optomechanical coupling in a two-dimensional photonic crystal defect cavity. Phys. Rev. Lett. 2011, 106, 203902. [CrossRef] [PubMed]

82. Winger, M.; Blasius, T.D.; Mayer Alegre, T.P.; Safavi-Naeini, A.H.; Meenehan, S.; Cohen, J.; Stobbe, S.; Painter, O. A chip-scale integrated cavity-electro-optomechanics platform. Opt. Express 2011, 19, 24905-24921. [CrossRef] [PubMed]

83. Zheng, J.; Sun, X.; Li, Y.; Poot, M.; Dadgar, A.; Shi, N.N.; Pernice, W.H.; Tang, H.X.; Wong, C.W. Femtogram dispersive L3-nanobeam optomechanical cavities: Design and experimental comparison. Opt. Express 2012, 20, 26486-26498. [CrossRef] [PubMed]

84. Antoni, T.; Kuhn, A.G.; Briant, T.; Cohadon, P.F.; Heidmann, A.; Braive, R.; Beveratos, A.; Abram, I.; Le Gratiet, L.; Sagnes, I.; et al. Deformable two-dimensional photonic crystal slab for cavity optomechanics. Opt. Lett. 2011, 36, 3434-3436. [CrossRef]

85. Safavi-Naeini, A.H.; Alegre, T.P.M.; Winger, M.; Painter, O. Optomechanics in an ultrahigh-Q two-dimensional photonic crystal cavity. Appl. Phys. Lett. 2010, 97, 181106. [CrossRef]

86. Sun, X.; Zheng, J.; Poot, M.; Wong, C.W.; Tang, H.X. Femtogram doubly clamped nanomechanical resonators embedded in a high-Q two-dimensional photonic crystal nanocavity. Nano Lett. 2012, 12, 2299-2305. [CrossRef]

87. Meade, R.; Winn, J.N.; Joannopoulos, J. Photonic Crystals: Molding the Flow of Light; Princeton University Press: Princeton, NJ, USA, 1995.

88. Faraon, A.; Waks, E.; Englund, D.; Fushman, I.; Vučković, J. Efficient photonic crystal cavity-waveguide couplers. Appl. Phys. Lett. 2007, 90, 073102. [CrossRef] 
89. Yang, D.; Tian, H.; Wu, N.; Yang, Y.; Ji, Y. Nanoscale torsion-free photonic crystal pressure sensor with ultra-high sensitivity based on side-coupled piston-type microcavity. Sens. Actuators A Phys. 2013, 199, 30-36. [CrossRef]

90. Talneau, A.; Mulot, M.; Anand, S.; Lalanne, P. Compound cavity measurement of transmission and reflection of a tapered single-line photonic-crystal waveguide. Appl. Phys. Lett. 2003, 82, 2577-2579. [CrossRef]

91. Johnson, S.G.; Ibanescu, M.; Skorobogatiy, M.; Weisberg, O.; Joannopoulos, J.; Fink, Y. Perturbation theory for Maxwell's equations with shifting material boundaries. Phys. Rev. E 2002, 65, 066611. [CrossRef] [PubMed]

92. Quan, Q.; Floyd, D.L.; Burgess, I.B.; Deotare, P.B.; Frank, I.W.; Tang, S.K.; Ilic, R.; Loncar, M. Single particle detection in CMOS compatible photonic crystal nanobeam cavities. Opt. Express 2013, 21, 32225-32233. [CrossRef] [PubMed]

93. Liang, F.; Quan, Q. Detecting single gold nanoparticles $(1.8 \mathrm{~nm})$ with ultrahigh-Q air-mode photonic crystal nanobeam cavities. ACS Photonics 2015, 2, 1692-1697. [CrossRef]

94. Lin, S.; Crozier, K.B. Trapping-assisted sensing of particles and proteins using on-chip optical microcavities. ACS Nano 2013, 7, 1725-1730. [CrossRef] [PubMed]

95. Li, Q.; Wang, T.; Su, Y.; Yan, M.; Qiu, M. Coupled mode theory analysis of mode-splitting in coupled cavity system. Opt. Express 2010, 18, 8367-8382. [CrossRef]

96. Jiang, W.; Mayor, F.M.; Patel, R.N.; McKenna, T.P.; Sarabalis, C.J.; Safavi-Naeini, A.H. Nanobenders: Efficient piezoelectric actuators for widely tunable nanophotonics at CMOS-level voltages. arXiv 2019, arXiv:1911.10973.

97. Lee, C.; Radhakrishnan, R.; Chen, C.-C.; Li, J.; Thillaigovindan, J.; Balasubramanian, N. Design and modeling of a nanomechanical sensor using silicon photonic crystals. J. Lightwave Technol. 2008, 26, 839-846. [CrossRef]

98. Tung, B.T.; Dao, D.V.; Ikeda, T.; Kanamori, Y.; Hane, K.; Sugiyama, S. Investigation of strain sensing effect in modified single-defect photonic crystal nanocavity. Opt. Express 2011, 19, 8821-8829. [CrossRef] [PubMed]

99. Tung, B.T.; Dao, D.V.; Ikeda, T.; Kanamori, Y.; Hane, K.; Sugiyama, S. Longitudinal strain sensitive effect in a photonic crystal cavity. Procedia Eng. 2011, 25, 1357-1360. [CrossRef]

100. Yang, Y.; Tian, H.; Yang, D.; Wu, N.; Zhou, J.; Liu, Q.; Ji, Y. Nanomechanical three dimensional force photonic crystal sensor using shoulder-coupled resonant cavity with an inserted pillar. Sens. Actuators A Phys. 2014, 209, 33-40. [CrossRef]

101. Qiao, Q.; Peng, C.; Xia, J.; Lee, C.; Zhou, G. Ultra-small photonic crystal (PhC)-based test tool for gas permeability of polymers. Opt. Express 2019, 27, 35600-35608. [CrossRef] [PubMed]

102. Petruzzella, M.; La China, F.; Intonti, F.; Caselli, N.; De Pas, M.; van Otten, F.; Gurioli, M.; Fiore, A. Nanoscale mechanical actuation and near-field read-out of photonic crystal molecules. Phys. Rev. B 2016, 94, 115413. [CrossRef]

103. Zobenica, Ž.; van der Heijden, R.; Petruzzella, M.; Pagliano, F.; Leijssen, R.; Xia, T.; Midolo, L.; Cotrufo, M.; Cho, Y.J.; van Otten, F. Fully integrated nano-opto-electro-mechanical wavelength and displacement sensor. In Proceedings of the Optical Sensors, Vancouver, BC, Canada, 18-20 July 2016; p. SeW2E.4.

104. Zhu, Y.; Lee, J.; Seshia, A. System-level simulation of a micromachined electrometer using a time-domain variable capacitor circuit model. J. Micromech. Microeng. 2007, 17, 1059. [CrossRef]

105. Rosenberg, J.; Lin, Q.; Painter, O. Static and dynamic wavelength routing via the gradient optical force. Nat. Photonics 2009, 3, 478-483. [CrossRef]

106. Berman, P.R.; Lin, C.C.; Arimondo, E. Advances in Atomic, Molecular, and Optical Physics; Elsevier: Amsterdam, The Netherlands, 2006.

107. Wiederhecker, G.S.; Chen, L.; Gondarenko, A.; Lipson, M. Controlling photonic structures using optical forces. Nature 2009, 462, 633-636. [CrossRef] [PubMed]

108. Yu, W.; Jiang, W.C.; Lin, Q.; Lu, T. Cavity optomechanical spring sensing of single molecules. Nat. Commun. 2016, 7, 12311. [CrossRef]

109. Pan, F.; Cui, K.; Bai, G.; Feng, X.; Liu, F.; Zhang, W.; Huang, Y. Radiation-Pressure-Antidamping Enhanced Optomechanical Spring Sensing. ACS Photonics 2018, 5, 4164-4169. [CrossRef]

110. Black, E.D. An introduction to Pound-Drever-Hall laser frequency stabilization. Am. J. Phys. 2001, 69, 79-87. [CrossRef]

111. Anetsberger, G.; Gavartin, E.; Arcizet, O.; Unterreithmeier, Q.P.; Weig, E.M.; Gorodetsky, M.L.; Kotthaus, J.P.; Kippenberg, T.J. Measuring nanomechanical motion with an imprecision below the standard quantum limit. Phys. Rev. A 2010, 82, 061804. [CrossRef] 
112. Safavi-Naeini, A.H.; Groblacher, S.; Hill, J.T.; Chan, J.; Aspelmeyer, M.; Painter, O. Squeezed light from a silicon micromechanical resonator. Nature 2013, 500, 185-189. [CrossRef]

113. Khanaliloo, B.; Jayakumar, H.; Hryciw, A.C.; Lake, D.P.; Kaviani, H.; Barclay, P.E. Single-crystal diamond nanobeam waveguide optomechanics. Phys. Rev. X 2015, 5, 041051. [CrossRef]

114. Burek, M.J.; Chu, Y.; Liddy, M.S.; Patel, P.; Rochman, J.; Meesala, S.; Hong, W.; Quan, Q.; Lukin, M.D.; Loncar, M. High quality-factor optical nanocavities in bulk single-crystal diamond. Nat. Commun. 2014, 5, 5718. [CrossRef] [PubMed]

115. Lu, X.; Lee, J.Y.; Lin, Q. Silicon carbide zipper photonic crystal optomechanical cavities. Appl. Phys. Lett. 2020, 116, 221104. [CrossRef] [PubMed]

116. Lee, J.Y.; Lu, X.; Lin, Q. High-Q silicon carbide photonic-crystal cavities. Appl. Phys. Lett. 2015, 106, 041106. [CrossRef]

117. Kaviani, H.; Healey, C.; Wu, M.; Ghobadi, R.; Hryciw, A.; Barclay, P.E. Nonlinear optomechanical paddle nanocavities. Optica 2015, 2, 271-274. [CrossRef]

118. Healey, C.; Kaviani, H.; Wu, M.; Khanaliloo, B.; Mitchell, M.; Hryciw, A.C.; Barclay, P.E. Design and experimental demonstration of optomechanical paddle nanocavities. Appl. Phys. Lett. 2015, 107, 231107. [CrossRef]

119. Healey, C.; Kaviani, H.; Barclay, P.E. Air mode silicon nitride photonic crystals and their application to nonlinear quantum optomechanical sensing. arXiv 2019, arXiv:1905.03341.

120. Paraïso, T.K.; Kalaee, M.; Zang, L.; Pfeifer, H.; Marquardt, F.; Painter, O. Position-squared coupling in a tunable photonic crystal optomechanical cavity. Phys. Rev. X 2015, 5, 041024. [CrossRef]

121. Doolin, C.; Hauer, B.; Kim, P.; MacDonald, A.; Ramp, H.; Davis, J. Nonlinear optomechanics in the stationary regime. Phys. Rev. A 2014, 89, 053838. [CrossRef]

122. Luan, X.; Huang, Y.; Li, Y.; McMillan, J.F.; Zheng, J.; Huang, S.W.; Hsieh, P.C.; Gu, T.; Wang, D.; Hati, A.; et al. An integrated low phase noise radiation-pressure-driven optomechanical oscillator chipset. Sci. Rep. 2014, 4, 6842. [CrossRef] [PubMed]

123. Sun, X.; Zhang, X.; Poot, M.; Xiong, C.; Tang, H.X. A superhigh-frequency optoelectromechanical system based on a slotted photonic crystal cavity. Appl. Phys. Lett. 2012, 101, 221116. [CrossRef]

124. Wu, M.; Hryciw, A.C.; Healey, C.; Lake, D.P.; Jayakumar, H.; Freeman, M.R.; Davis, J.P.; Barclay, P.E. Dissipative and dispersive optomechanics in a nanocavity torque sensor. Phys. Rev. X 2014, 4, 021052. [CrossRef]

125. Hajisalem, G.; Losby, J.E.; de Oliveira Luiz, G.; Sauer, V.T.; Barclay, P.E.; Freeman, M.R. Two-axis cavity optomechanical torque characterization of magnetic microstructures. New J. Phys. 2019, 21, 095005. [CrossRef]

126. Wu, M.; Hryciw, A.C.; Khanaliloo, B.; Freeman, M.R.; Davis, J.P.; Barclay, P.E. Photonic crystal paddle nanocavities for optomechanical torsion sensing. In Proceedings of the CLEO: Science and Innovations, San Jose, CA, USA, 6-11 October 2012; p. CW1M.7.

127. Li, H.; Li, M. Optomechanical photon shuttling between photonic cavities. Nat. Nanotechnol. 2014, 9, 913-919. [CrossRef]

128. Kim, P.H.; Doolin, C.; Hauer, B.D.; MacDonald, A.J.; Freeman, M.R.; Barclay, P.E.; Davis, J.P. Nanoscale torsional optomechanics. Appl. Phys. Lett. 2013, 102, 053102. [CrossRef]

129. Burgess, J.A.; Fraser, A.E.; Sani, F.F.; Vick, D.; Hauer, B.D.; Davis, J.P.; Freeman, M.R. Quantitative magneto-mechanical detection and control of the Barkhausen effect. Science 2013, 339, 1051-1054. [CrossRef]

130. Krause, A.G.; Winger, M.; Blasius, T.D.; Lin, Q.; Painter, O. A high-resolution microchip optomechanical accelerometer. Nat. Photonics 2012, 6, 768-772. [CrossRef]

131. Liu, F.; Hossein-Zadeh, M. Mass sensing with optomechanical oscillation. IEEE Sens. J. 2012, 13, $146-147$. [CrossRef]

132. Chen, Z.; Wu, X.; Liu, L.; Xu, L. Optical Spring Effect in Micro-Bubble Resonators and Its Application for the Effective Mass Measurement of Optomechanical Resonant Mode. Sensors 2017, 17, 2256. [CrossRef] [PubMed]

133. Maksymowych, M.; Westwood-Bachman, J.; Venkatasubramanian, A.; Hiebert, W. Optomechanical spring enhanced mass sensing. Appl. Phys. Lett. 2019, 115, 101103. [CrossRef]

134. Ren, L.; Li, Y.; Li, N.; Chen, C. Trapping and Optomechanical Sensing of Particles with a Nanobeam Photonic rystal Cavity. Crystals 2019, 9, 57. [CrossRef] 
135. Cui, K.; Huang, Z.; Xu, Q.; Pan, F.; Xiong, J.; Feng, X.; Liu, F.; Zhang, W.; Huang, Y. Phonon-laser sensing in a hetero optomechanical crystal cavity. arXiv 2019, arXiv:1906.12057.

136. Zhang, Y.; Ai, J.; Xiang, Y.; He, Q.; Li, T.; Ma, J.I. Mass sensor based on split-nanobeam optomechanical oscillator. In Selected Papers of the Chinese Society for Optical Engineering Conferences Held October and November; International Society for Optics and Photonics: Bellingham, WA, USA, 2017; p. 102552U.

137. Zhang, H.; Zhao, X.; Wang, Y.; Huang, Q.; Xia, J. Femtogram scale high frequency nano-optomechanical resonators in water. Opt. Express 2017, 25, 821-830. [CrossRef]

138. Zhang, H.; Zeng, C.; Chen, D.; Li, M.; Wang, Y.; Huang, Q.; Xiao, X.; Xia, J. Femtogram scale nanomechanical resonators embedded in a double-slot photonic crystal nanobeam cavity. Appl. Phys. Lett. 2016, 108, 051106. [CrossRef]

139. Miao, T.; Xiao, D.; Li, Q.; Hou, Z.; Wu, X. A 4 mm2 Double Differential Torsional MEMS Accelerometer Based on a Double-Beam Configuration. Sensors 2017, 17, 2264. [CrossRef]

140. Ciotirca, L.E.; Bernal, O.; Enjalbert, J.; Cassagnes, T.; Tap, H.; Beaulaton, H.; Şahin, S. New Stability Method of a Multirate Controller for a Three-Axis High-\$ Q \$ MEMS Accelerometer With Simultaneous Electrostatic Damping. IEEE Sens. J. 2018, 18, 6106-6114. [CrossRef]

141. Lam, T.T.; Gagliardi, G.; Salza, M.; Chow, J.H.; De Natale, P. Optical fiber three-axis accelerometer based on lasers locked to $\pi$ phase-shifted Bragg gratings. Meas. Sci. Technol. 2010, 21, 094010. [CrossRef]

142. Cervantes, F.G.; Kumanchik, L.; Pratt, J.; Taylor, J. Self-calibrating ultra-low noise, wide-bandwidth optomechanical accelerometer. Appl. Phys. Lett. 2014, 104, 221111.

143. Huang, Y.; Flor Flores, J.G.; Li, Y.; Wang, W.; Wang, D.; Goldberg, N.; Zheng, J.; Yu, M.; Lu, M.; Kutzer, M. A Chip-Scale Oscillation-Mode Optomechanical Inertial Sensor Near the Thermodynamical Limits. Laser Photonics Rev. 2020, 14, 1800329. [CrossRef]

144. Wong, C.W.; Li, Y.; Zheng, J.; Rogers, D. Apparatus for Measuring Gravitational Force and Methods of Using the Same. U.S. Patent Application No. 13/587,689, 21 February 2013.

145. Wong, C.W.; Li, Y.; Zheng, J.; Rogers, D.J. Chip-Scale Optomechanical Gravimeter. U.S. Patent No. 8,867,0262014, 21 August 2014.

146. He, L.; Li, H.; Li, M. Optomechanical measurement of photon spin angular momentum and optical torque in integrated photonic devices. Sci. Adv. 2016, 2, e1600485. [CrossRef]

147. Kaviani, H.; Ghobadi, R.; Behera, B.; Wu, M.; Hryciw, A.; Vo, S.; Fattal, D.; Barclay, P. Optomechanical detection of light with orbital angular momentum. Opt. Express 2020, 28, 15482-15496. [CrossRef] [PubMed]

148. Suzuki, H.; Brown, E.; Sterling, R. Nonlinear dynamics of an optomechanical system with a coherent mechanical pump: Second-order sideband generation. Phys. Rev. A 2015, 92, 033823. [CrossRef]

149. Wang, C.; Chen, H.J.; Zhu, K.D. Nonlinear optical response of cavity optomechanical system with second-order coupling. Appl. Opt. 2015, 54, 4623-4628. [CrossRef]

150. Djorwé, P.; Mbé, J.H.T.; Engo, S.G.N.; Woafo, P. Classical and semiclassical studies of nonlinear nano-optomechanical oscillators. Eur. Phys. J. D 2013, 67, 45. [CrossRef]

151. Li, H.; Chen, Y.; Noh, J.; Tadesse, S.; Li, M. Multichannel cavity optomechanics for all-optical amplification of radio frequency signals. Nat. Commun. 2012, 3, 1091. [CrossRef]

152. Brawley, G.A.; Vanner, M.R.; Larsen, P.E.; Schmid, S.; Boisen, A.; Bowen, W.P. Nonlinear optomechanical measurement of mechanical motion. Nat. Commun. 2016, 7, 10988. [CrossRef]

153. Leijssen, R.; La Gala, G.R.; Freisem, L.; Muhonen, J.T.; Verhagen, E. Nonlinear cavity optomechanics with nanomechanical thermal fluctuations. Nat. Commun. 2017, 8, ncomms16024. [CrossRef]

154. Navarro-Urrios, D.; Capuj, N.E.; Colombano, M.F.; Garcia, P.D.; Sledzinska, M.; Alzina, F.; Griol, A.; Martinez, A.; Sotomayor-Torres, C.M. Nonlinear dynamics and chaos in an optomechanical beam. Nat. Commun. 2017, 8, 14965. [CrossRef] [PubMed]

155. Eichler, A.; Moser, J.; Chaste, J.; Zdrojek, M.; Wilson-Rae, I.; Bachtold, A. Nonlinear damping in mechanical resonators made from carbon nanotubes and graphene. Nat. Nanotechnol. 2011, 6, 339-342. [CrossRef] [PubMed]

156. Singh, V.; Shevchuk, O.; Blanter, Y.M.; Steele, G.A. Negative nonlinear damping of a graphene mechanical resonator. arXiv 2015, arXiv:1508.04298. [CrossRef]

157. Shevchuk, O.; Singh, V.; Steele, G.A.; Blanter, Y.M. Optomechanical response of a nonlinear mechanical resonator. Phys. Rev. B 2015, 92, 195415. [CrossRef] 
158. Weis, S.; Rivière, R.; Deléglise, S.; Gavartin, E.; Arcizet, O.; Schliesser, A.; Kippenberg, T.J. Optomechanically induced transparency. Science 2010, 330, 1520-1523. [CrossRef]

159. Xiong, H.; Wu, Y. Fundamentals and applications of optomechanically induced transparency. Appl. Phys. Rev. 2018, 5, 031305. [CrossRef]

160. Fan, J.; Huang, C.; Zhu, L. Optomechanical nonlinearity enhanced optical sensors. Opt. Express 2015, 23, 2973-2981. [CrossRef]

161. Liu, Z.X.; Wang, B.; Kong, C.; Si, L.G.; Xiong, H.; Wu, Y. A proposed method to measure weak magnetic field based on a hybrid optomechanical system. Sci. Rep. 2017, 7, 12521. [CrossRef]

162. Liu, Z.-X. Precision measurement of magnetic field based on second-order sideband generation in a hybrid electromagnetic-optomechanical system. IEEE Sens. J. 2018, 18, 9145-9150. [CrossRef]

163. Zhang, J.-Q.; Li, Y.; Feng, M.; Xu, Y. Precision measurement of electrical charge with optomechanically induced transparency. Phys. Rev. A 2012, 86, 053806. [CrossRef]

164. Xiong, H.; Si, L.-G.; Wu, Y. Precision measurement of electrical charges in an optomechanical system beyond linearized dynamics. Appl. Phys. Lett. 2017, 110, 171102. [CrossRef]

165. Xiong, H.; Liu, Z.X.; Wu, Y. Highly sensitive optical sensor for precision measurement of electrical charges based on optomechanically induced difference-sideband generation. Opt. Lett. 2017, 42, 3630-3633. [CrossRef] [PubMed]

166. Liu, J.; Zhu, K. Enhanced sensing of millicharged particles using nonlinear effects in an optomechanical system. Opt. Express 2018, 26, 2054-2064. [CrossRef] [PubMed]

167. Li, L.; Yang, W.-X.; Zhang, Y.; Shui, T.; Chen, A.-X.; Jiang, Z. Enhanced generation of charge-dependent second-order sideband and high-sensitivity charge sensors in a gain-cavity-assisted optomechanical system. Phys. Rev. A 2018, 98, 063840. [CrossRef]

168. Du, H.; Chau, F.S.; Zhou, G. Harmonically-Driven Snapping of a Micromachined Bistable Mechanism With Ultra-Small Actuation Stroke. J. Microelectromech. Syst. 2018, 27, 34-39. [CrossRef]

169. Bhimanapati, G.R.; Lin, Z.; Meunier, V.; Jung, Y.; Cha, J.; Das, S.; Xiao, D.; Son, Y.; Strano, M.S.; Cooper, V.R.; et al. Recent Advances in Two-Dimensional Materials beyond Graphene. ACS Nano 2015, 9, 11509-11539. [CrossRef] [PubMed]

170. Bonaccorso, F.; Sun, Z.; Hasan, T.; Ferrari, A. Graphene photonics and optoelectronics. Nat. Photonics 2010, 4, 611-622. [CrossRef]

171. Bao, Q.; Loh, K.P. Graphene photonics, plasmonics, and broadband optoelectronic devices. ACS Nano 2012, 6, 3677-3694. [CrossRef]

172. Naumis, G.G.; Barraza-Lopez, S.; Oliva-Leyva, M.; Terrones, H. Electronic and optical properties of strained graphene and other strained 2D materials: A review. Rep. Prog. Phys. 2017, 80, 096501. [CrossRef]

173. Yu, L.; Yin, Y.; Shi, Y.; Dai, D.; He, S. Thermally tunable silicon photonic microdisk resonator with transparent graphene nanoheaters. Optica 2016, 3, 159-166. [CrossRef]

174. Zangeneh-Nejad, F.; Safian, R. A graphene-based THz ring resonator for label-free sensing. IEEE Sens. J. 2016, 16, 4338-4344. [CrossRef]

175. Wang, X.; Cui, Y.; Li, T.; Lei, M.; Li, J.; Wei, Z. Recent advances in the functional 2D photonic and optoelectronic devices. Adv. Opt. Mater. 2019, 7, 1801274. [CrossRef]

176. Gan, X.; Shiue, R.J.; Gao, Y.; Mak, K.F.; Yao, X.; Li, L.; Szep, A.; Walker, D., Jr.; Hone, J.; Heinz, T.F.; et al. High-contrast electrooptic modulation of a photonic crystal nanocavity by electrical gating of graphene. Nano Lett. 2013, 13, 691-696. [CrossRef]

177. Williamson, I.A.; Mousavi, S.H.; Wang, Z. Large cavity-optomechanical coupling with graphene at infrared and terahertz frequencies. ACS Photonics 2016, 3, 2353-2361. [CrossRef]

178. De Alba, R.; Massel, F.; Storch, I.R.; Abhilash, T.S.; Hui, A.; McEuen, P.L.; Craighead, H.G.; Parpia, J.M. Tunable phonon-cavity coupling in graphene membranes. Nat. Nanotechnol. 2016, 11, 741-746. [CrossRef]

179. Liu, M.; Yin, X.; Ulin-Avila, E.; Geng, B.; Zentgraf, T.; Ju, L.; Wang, F.; Zhang, X. A graphene-based broadband optical modulator. Nature 2011, 474, 64-67. [CrossRef]

180. Singh, V.; Sengupta, S.; Solanki, H.S.; Dhall, R.; Allain, A.; Dhara, S.; Pant, P.; Deshmukh, M.M. Probing thermal expansion of graphene and modal dispersion at low-temperature using graphene nanoelectromechanical systems resonators. Nanotechnology 2010, 21, 165204. [CrossRef]

181. Guizal, B.; Antezza, M. Light-induced optomechanical forces in graphene waveguides. Phys. Rev. B 2016, 93, 115427. [CrossRef] 
182. Xu, Z.; Qiu, C.; Yang, Y.; Zhu, Q.; Jiang, X.; Zhang, Y.; Gao, W.; Su, Y. Ultra-compact tunable silicon nanobeam cavity with an energy-efficient graphene micro-heater. Opt. Express 2017, 25, 19479-19486. [CrossRef]

183. Fang, K.; Matheny, M.H.; Luan, X.; Painter, O. Optical transduction and routing of microwave phonons in cavity-optomechanical circuits. Nat. Photonics 2016, 10, 489-496. [CrossRef]

184. Xu, X.-W.; Li, Y.; Chen, A.-X.; Liu, Y.-X. Nonreciprocal conversion between microwave and optical photons in electro-optomechanical systems. Phys. Rev. A 2016, 93, 023827. [CrossRef]

185. Zhu, J.; Zhao, G.; Savukov, I.; Yang, L. Polymer encapsulated microcavity optomechanical magnetometer. Sci. Rep. 2017, 7, 8896. [CrossRef] [PubMed]

186. Yuan, Y.; Chen, W.; Ma, Z.; Deng, Y.; Chen, Y.; Chen, Y.; Hu, W. Enhanced optomechanical properties of mechanochemiluminescent poly(methyl acrylate) composites with granulated fluorescent conjugated microporous polymer fillers. Chem. Sci. 2019, 10, 2206-2211. [CrossRef] [PubMed]

C 2020 by the authors. Licensee MDPI, Basel, Switzerland. This article is an open access article distributed under the terms and conditions of the Creative Commons Attribution (CC BY) license (http://creativecommons.org/licenses/by/4.0/). 